\title{
Über einige anatomische Ähnlichkeiten zwischen progressiver Paralyse und multipler Sklerose.
}

(Untersuchungen über herdförmigen Markfaserschwund bei Paralyse.)

\author{
Von \\ W. Spielmeyer.
}

(Aus der Psychiatrischen und Nervenklinik in Freiburg i. Br. [Prof. Dr. Hoche].)

Mit 7 Textfiguren und 7 Tafeln.

(Eingegangen am 17. Mai 1910.)

Das Interesse an den Markscheidenbilder $\mathrm{n}$ bei der progressiven Paralyse, das nach den Mitteilungen Tuczeks und nach der Weigertschen Entdeckung einer sicher arbeitenden Markscheidenmethode im Mittelpunkte der anatomischen Paralyseforschung gestanden hatte, ist besonders seit dem Ende der 90er Jahre in den Hintergrund getreten. Es hatte sich gezeigt, daß ein Schwund der Rindenfasern, zumal in ihren oberen Geflechten, auch von anderen organischen Rindenkrankheiten verursacht wird, so daß eine anatomische Diagnose der Paralyse auf Grund des Markfaserbildes allein in einwandfreier Weise nicht möglich schien. Auch die genaue Ermittlung, wie die verschiedenen Markfaserschichten der Rinde zeitlich und graduell von dem paralytischen Prozeß getroffen werden, konnte in differentialdiagnostischer Hinsicht keine sicheren Anhaltspunkte geben, obschon solche Untersuchungen, ebenso wie das Studium der Ausbreitung des Markfaserschwundes über die verschiedenen Regionen des Hemisphärenhirnes, für die Kenntnis des Prozesses ihre Bedeutung besitzen.

Für die Differentialdiagnose der Paralyse aber ergaben sich die wesentlichsten Merkmale aus den Bildern der Nißlschen AlkoholSeifen-Methylenblaufärbung, welche die Nerven- und Gliazellen und auch die mesodermalen Elemente in den Gefäßwänden und den Meningen zur Darstellung bringt. Die grundlegenden Untersuchungen, die uns an den $\mathrm{Ni}$ Blschen Zellbildern und an den Weigertschen Neurogliapräparaten die anatomische Diagnose der progressiven Paralyse gelehrt haben, sind jedem bekannt. Die Fortschritte, die sie in der Erkenntnis der pathologischen Veränderungen bei der paralytischen Hirnerkrankung brachten, ließen es von vornherein lohnender 
erscheinen, mit diesen Methoden zu arbeiten, als die Ausfälle in der kortikalen Markfaserung bei der Paralyse zu studieren.

Eine Belebung des Interesses an den Markfaserbildern von der paralytischen Rinde brachte dann die Entdeckung der Methoden, welche die marklosen Fasern und Fibrillen darstellen. An paralytischen Rinden zeigte sich nicht selten ein merkwürdiger Unterschied zwischen erheblichen Lichtungen im Weigertschen Markscheidenpräparat und einem scheinbar normalen Faserreichtum im Bielschowskyschen Achsenzylinderpräparat (Bielschowsky und Brodmann, Renkichi Moriasu u. a.). Es ließ sich dieser Kontrast nicht etwa allein daraus erklären, daß sich in dem ungemein dichten Gewirr von Fasern im Silberpräparat die Ausfälle leicht dem Nachweise entziehen; sondern man mußte auch annehmen, daß eine Persistenz nackter Fasern in der paralytischen Rinde vorkomme. Diese Befunde gaben so eine Bestätigung für die früher von Alzheimer und Kaes ausgesprochene Vermutung, daß eine Erkrankung der Markfasern sich mit dem Schwunde der Markhülle einleiten könne. Nach Kaes erklären sich die sog. ,gelichteten Faserschichten“ daraus, daß die Achsenzylinder bis auf ein Minimum ihres Markes beraubt, noch längere Zeit ihre Existenz fortführen. Und Alzhei mer ${ }^{1}$ ) meint, daß die mangelhafte Färbbarkeit der Markscheide auf eine Erkrankung der Markfaser hinwist, die dem Zerfall voraufgehe.

Für eine derartige Schädigung der Markhülle sprechen vor allem Bilder von solchen Windungen, in denen der Markfaserschwund nicht diffus, sondern unregelmäßig verteilt, fleckig ist. Man kann da oft sehen, wie die Fasern, ihres Markes mehr oder weniger völlig beraubt, in eine Lichtung eintreten und beim Verlassen derselben häufig wieder vom Mark umgeben sind; in den Marklichtungen scheinen also markarme und marklose Fasern zu persistieren. Sicheren Aufschluß darüber kann natürlich auch hier erst das Achsenzylinderpräparat geben; daß die Dinge tatsächlich so liegen, wie es das Markfaserpräparat bereits vermuten läßt, haben bekanntlich besonders Borda und Fischer gezeigt. Sie haben überhaupt erst von dem herdförmigen Markschwund, von welchem im Gegensatz zu dem diffusen Markausfall in der Rinde auffallend wenig die Rede war, eine ausführlichere Beschreibung gegeben.

Ich habe mich bei meinen Paralyseuntersuchungen ebenfalls mit den Eigentümlichkeiten des herdförmigen Markscheidenschwundes befaßt und dabei im wesentlichen feststellen können, was Fis ch er davon gesagt und abgebildet hat. Wenn ich mich heute zu diesem Thema äußere,

1) Alzheimer, Histologische Studien zur Differentialdiagnose der progressiven Paralyse. Histologische und histopathologische Arbeiten über die Großhinrinde. I. S. 62. 
so geschieht das, weil einige seltenere Bilder, die ich in der letzten Zeit fand, ein besonderes Interesse besitzen und vielleicht auch für die histologische Auffassung von den markarmen Herden nicht ohne Bedeutung sein dürften.

Ein besonderes Interesse schien mir der fleckige Markfaserschw und mit Rücksicht auf manche Ähnlichkeit mit Rindenherden der multiplen Sklerose zu besitzen. A uf eine Vergleichung des fleckigen Markscheidenschwundes mit den Rindenherden bei der multiplen Sklerose waren deshalb die Untersuchungen, von denen hier die Rede sein soll, vorwiegend gerichtet. Diese Mitteilung wird von dem fleckigen Markscheidenschwund in seiner Bedeutung für das histologische Gesamtbild der Paralyseundinseinen BeziehungenzudenSklerosenherden handeln.

An die Spitze der Erörterungen möchte ich einen Fall stellen, der mir für die Beurteilung der Frage von dem fleckigen Markfaserschwunde eine besondere Wichtigkeit zu besitzen scheint und der für diese Untersuchungen in vielen Beziehungen richtunggebend war.

Klinisch lag der Fall (D.) sehr einfach. Ein 44jähriger Mann erkrankte mit einer leichten Erregung, in welcher er durch seine Reizbarkeit, Unternehmungslust und besonders durch seine von vornherein unsinnigen Größenideen auffiel. Der Größenwahn steigerte sich rasch, ebenso seine Unruhe, und etwa ein halbes Jahr nach dem ersten Auftreten psychischer Störungen wurde seine Aufnahme in eine Anstalt notwendig. In der Klinik fiel außer dem blühenden Größenwahn eine allgemeine große. Urteils- und Gedächtnisschwäche auf. Von körperlich nervösen Zeichen bot er reflektorische Starre mit Differenz der Pupillen bei prompter Konvergenzreaktion, Fehlen der Patellarreflexe, Rombergsches Phänomen. Die anfängliche Erregung ging bald zurück, und es entwickelte sich in allmählich zunehmendem Maße das Bild apathischen Schwachsinns, in welchem auch die Größenideen abblaßten. Dieser Zustand von Stumpfheit und euphorischem Schwachsinn war bereits 1 Jahr nach dem Ausbruch der ersten psychischen Störungen erreicht und blieb für eine Reihe von Monaten gleich. Später wurde der Kranke für 2 bis 3 Monate wieder etwas lebhafter; er war öfters depressiv, verdrossen, später wieder, wie anfangs, leicht heiter erregt und glücklich in seinen schwachsinnigen Größenideen; dann wurde er wieder stumpf, hatte aber seine Tage, wo er an den Vorgängen der Umgebung Anteil nahm. Allmählich jedoch vertiefte sich die Teilnahmlosigkeit; apathisch und blöde $a B$ und rauchte er den ganzen Tag, verhielt sich Aufforderungen und Fragen gegenüber meist völlig reaktionslos, war im höchsten Maße unsauber usw. Während des letzten Jahres (vor seinem Tode) war sein Zustand unverändert der eines tief Verblödeten. Allmählich traten bei dem anfangs sehr kräftigen Menschen körperliche Schwächeerscheinungen auf; das mit der Vertiefung des Blödsinns stark angestiegene Gewicht nahm ab; es entwickelte sich Decubitus. Gegen Ende traten bisweilen nachts Zustände lebhafter motorischer Unruhe auf. Der neurologische Befund hatte sich nicht wesentlich geändert, nur waren noch ausgesprochene Sprachstörungen (verwaschene, tremulierende Sprache mit Silbenstolpern) hinzugekommen; die Hinterstrangsymptome bestanden in gleicher Weise fort; irgendwelche spastischen Erscheinungen wurden nie be- 
obachtet, ebenso auch keine Anfälle. 31/2 Jahre nach dem Beginne der Erkrankung (3 Jahre nach der Aufnahme in die Klinik) starb der Kranke, ohne irgendeine inter* kurrente Erkrankung.

Die anatomische Untersuchung bestätigte die Diagnose einer Paralyse.

Am Rückenmarke fand sich, entsprechend den früh einsetzenden und eindeutigen Hinterstrangsymptomen, die zu erwartende Degeneration in den Hinterw urzelbahnen. Die Hinterwurzelerkrankung betraf lokal vorwiegend die Sakral- und Lumbalsegmente und in geringerem Grade auch vereinzelte dorsale Segmente. Aufsteigend ließ sich von dort aus in den langen Hinterstrangbahnen eine deutliche

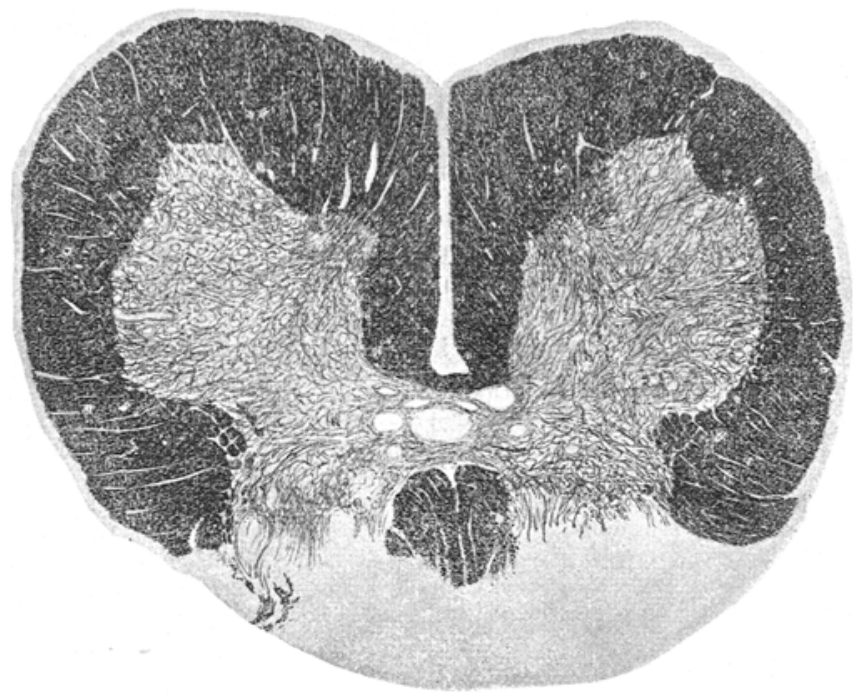

Fig. 1. Mittleres Lendenmark. Sklerotischer Hauptherd.

Degeneration verfolgen. Der Prozeß in den Hinterwurzeln hatte besonders auch die kurzen Bahnen betroffen, speziell die nach den untersten Abschnitten der Clarkeschen Säule strebenden Wurzelzüge, was sich in einer Lichtung der die Clarkesche Säulen hinten und medial umgebenden und sie durchsetzenden Markfasergeflechte kundgibt.

Außer der systematischen Hinterwurzeldegeneration aber fanden sich in der unteren Hälfte des Rückenmarks drei Herde, die im Weigert-Präparat durchaus denen bei multipler Sklerose entsprechen. Der größte Herd (Fig. 1) liegt im zweiten Lumbalsegment; er hat im Rückenmarksquerschnitt die Ausdehnung, wie sie die Figur wiedergibt, d. h. er nimmt etwa die hinteren zwei Drittel des Hinterstranges ein, überschreitet auf der einen Seite das Hinterhorn bis in den angrenzenden Seitenstrang hinein, auf der 
andern Seite endigt er mitten im Hinterhorn. Er ist scharf aus dem Mark herausgeschnitten, die Fasern der Hinterwurzeln sind wie abgebrochen, eine Strecke ihres Verlaufs erscheint ausgeschaltet; einige von den Hinterwurzelzügen setzen sich noch ein Stück weit in den Herd fort, und es liegen auch mitten in dem sonst total entfärbten Herde vereinzelte Wurzelbündel als schwarze Striche und Büschel. Eine weitere Besprechung des Herdes ist wohl unnötig. Die Fig. 1 zeigt, ebenso wie die folgende, was an den Markscheidenpräparaten zu sehen ist. Fig. 2 gibt ein Bild von dem ebenfalls hinten im Rückenmarksquerschnitt gelegenen zweiten Herde, der vor allem die Gegend des Hinterhorns einnimmt und darüber hinaus in das Seiten- und Hinterstrang-

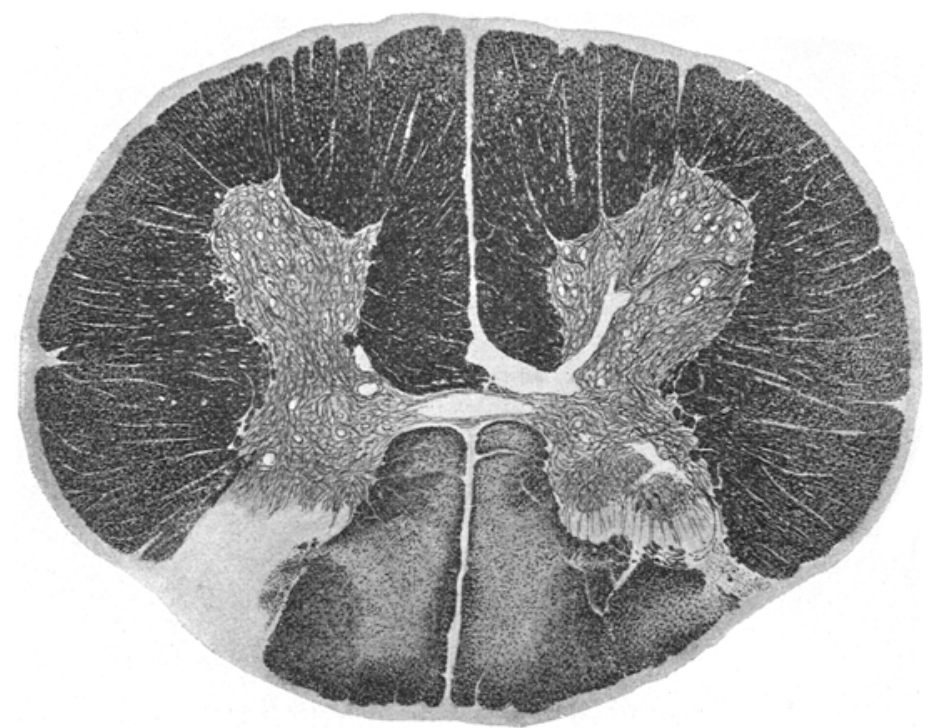

Fig. 2. Oberes Iendenmark. Kleiner Herd im Berelohe eines Hinterhornes. Hinterstrangdegeneration in den Wurzelarealen.

areal hineingreift. Der zweite Herd liegt etwa an der Grenze zwischen dem letzten Brust- und dem ersten Lendenmarksegment. Zwischen ihm und dem Hauptherd in L 2 ist eine herdfreie Partie, die ausschließlich die typische Figur der Hinterstrangdegenerationen in symmetrischer Weise zeigt: geringe lokale Degenerationen in der Wurzeleintrittszone und aufsteigende Degenerationen in den medialen Partien der Hinterstränge unter Freilassung der endogenen Areale. Schließlich fand sich noch ein dritter, wieder etwas größerer Herd in dem ersten Sakralsegmente, der ähnlich wie der Hauptherd gelegen, doch nicht ganz so ausgedehnt ist, d. h. nicht so weit nach vorn im Hinterstrang reicht. Auch zwischen diesem und dem Hauptherd liegen herdfreie Segmente. 
Es braucht nach den Markscheidenbildern wohl kaum weiter begründet zu werden, daß die Herde den echten Skleroseplaques gleichen: sie haben das Regellose, von Fasersystemen und Gefäßversorgung Unabhängige wie diese, sie sind in ihren Grenzen im allgemeinen scharf bestimmt, die an die Herdgrenze heranziehenden Fasern erscheinen wie abgeschnitten. Weiter sehen wir an den Markscheidenpräparaten, daß sich die Herde um die Degenerationsfelder der sensiblen Wurzeln in den Hintersträngen nicht ,,kümmern", sie beeinflussen auch die Degenerationsfigur in keiner Weise: die Herde haben, wie man an Fig. 3 sieht, keine sekundäre Degeneration zur Folge, das Feld der sekundären Degeneration ist ganz symmetrisch und hält sich an die betreffenden Wurzelareale.

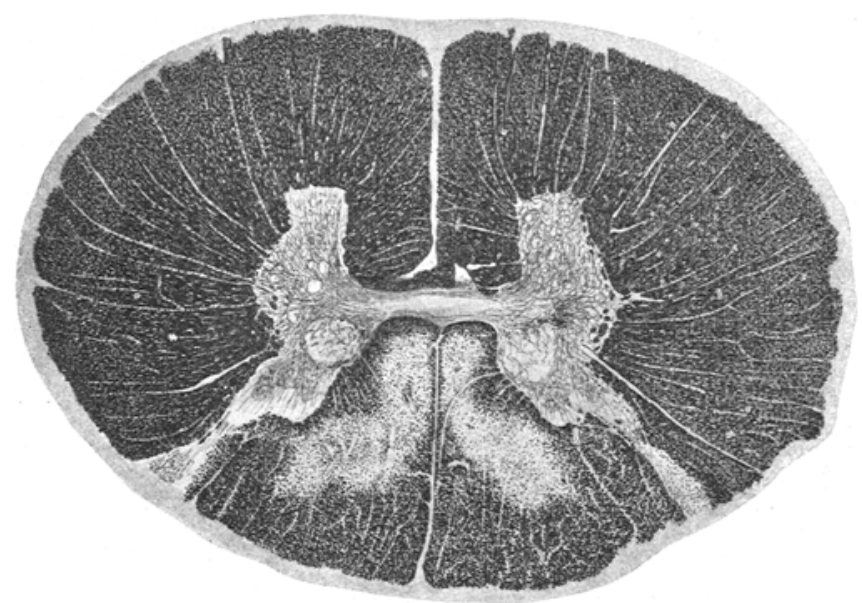

Fig. 3. Unteres Dorsalmark. Aufsteigende Hinterstrangdegeneration in den Wurzelarealen.

Es überrascht deshalb nicht, daß auch am Bielschowsky - Präparat die Ubereinstimmung der Bilder hier mit denen bei multipler Sklerose hervortritt; die Achsenzylinder sind in den Herden nicht nachweisbar an Zahl reduziert. Wo der Herd nur oder vorwiegend auf einer Seite gelegen ist, kann man sich besonders gut davon überzeugen, daß ein Unterschied zwischen der einen und der andern Seite im Verhalten der Achsenzylinder nicht besteht. Manchmal erkennt man die Gegend des Herdes an der nicht völlig entfärbten, krümelig schmutzigen Grundsubstanz.

Schließlich zeigt sich bei der Weigertschen Neurogliafärbung eine dichtfaserige, sehr kernarme Gliamasse. Da die Fasern, wie bei der multiplen Sklerose und vielen anderen gliösen Wucherungen in den Rückenmarkssträngen, sich vornehmlich zur Längsachse des Rücken- 
marks parallel stellen, also in gleicher Richtung wie die Strangfasern laufen, so sieht man auch hier am Querschnitt nur die häkchenförmig umgebogenen sehr feinen Faserquerschnitte, die außerordentlich dicht beisammen stehen. - Am Zellpräparate bemerkt man regressive Zellund Kernveränderungen an den Gliaelementen, geschrumpfte, oft dunkelgefärbte Kerne und zackig hervorspringende, ebenfalls etwas scharf gefärbte Zellfortsätze.

So ist also die Eigenart der Herde wohl bestimmt. Sie gleichen durchaus denen bei der multiplen Sklerose. Irgendwelche anderen plaqueförmigen Sklerosen kommen bei einem Vergleiche nicht in Betracht. Von Erweichungs- oder Entzündungsherden kann keine Rede sein; sie erweisen sich auch absolut unabhängig von regressiven Gefäßveränderungen; die infiltrativen Vorgänge, welche wir im Rückenmark, wie sonst bei Paralyse, an den spinalen Gefäßen und besonders an der Pia finden, sind in den Herden und ihrer Umgebung keineswegs stärker als sonst.

Wir haben natürlich möglichst alle Segmente des Rückenmarks nach solchen Herden durchsucht, aber weiter keine gefunden. Und auch die basalen Ganglien, der Hirnstam m und das Kleinhirn erwiesen sich überall frei von solchen.

Es steht nun die Frage zur Beantwortung: Handelt es sich hier um eine Kombination von multipler Sklerose und Paralyse? Oder kann die paralytische zentrale Erkrankung -- so ungewöhnlich dies wäre - a uch ein mal sklerotische Herde hervor bringen?

In der Literatur ist von den Beziehungen der multiplen Sklerose zur progressiven Paralyse früher häufiger die Rede gewesen. Man sprach von Misch- und Ubergangsformen, in welcher sogar die Entscheidung an der Hand des anatomischen Präparats nicht mit Sicherheit gelingen sollte. In der Diskussion über diese Misch- und Utbergangsformen haben immer die beiden Fälle von Schultze ${ }^{\mathbf{1}}$ ) und die Beobachtung von Zacher ${ }^{2}$ ) eine besondere Beachtung gefunden. Schultze suchte an zwei einschlägigen Fällen zu zeigen, daß anatomisch zwischen den genannten beiden Krankheitsformen nähere verwandtschaftliche Beziehungen bestehen. Beide hätten zur gemeinsamen Basis chronisch entzündliche Prozesse des Gefäßbindegewebsapparates im zentralen Nervensystem, welche in dem einen Falle sich mehr diffus, wenn auch an verschiedenen Stellen verschieden stark über das Zentralnervensystem verbreiten, in dem andern Falle aber

1) Über die Beziehungen der multiplen Sklerose des zentralen Nervensystems zur allgemeinen progressiven Paralyse der Irren. Archiv f. Psychiatrie 11, 216.

2) Ein Fall von sogenannter Misch- und Utbergangsform der progressiven Paralyse und der multiplen Herdsklerose. Archiv f. Psychiatrie 13, 168. 
herd- und fleckweise aufträten (vgl. Zacher l. c. S. 169). Die diffuse Sklerose bei der progressiven Paralyse könne eine Abart der multiplen sein.

Diese Beobachtungen von Schultze und Zacher liegen jetzt fast 30 Jahre zurück; inzwischen haben sich die Anschauungen von dem Wesen des paralytischen Prozesses und der multiplen Sklerose beträchtlich geändert; und je mehr man Paralyse und multiple Sklerose klinisch und vor allem anatomisch diagnostizieren lernte, desto seltener sind die Mitteilungen über solche UUbergangsformen der beiden Prozesse geworden. Petrof $f^{1}$ ), der in seiner aus der Jollysch enKlinik stammenden Dissertation das einschlägige Material zusammengetragen hat, fand 14 Fälle in der Literatur, von denen nur 6 pathologisch-anatomisch eine Mischform der multiplen Sklerose und der progressiven Paralyse reprüsentierten. In den übrigen 8 war entweder nur die Hirnrinde in Form multipler Herde oder das Mark in besonders ausgedehnter Weise erkrankt, oder es lag eine diffuse Sklerose in Rinde und Mark vor. Von dieser zweiten Gruppe der von Petroff zusammengestellten Fälle sagt Seiffer ${ }^{2}$ ), daß sie klinisch ebenso ungleichartig sind, wie anatomisch, und daß ihre Zugehörigkeit zur multiplen Sklerose teilweise mehr als zweifelhaft ist.

Der Frage nach den sog. Misch- und Ǔbergangsfällen heute auf Grund der Literatur nachzugehen, erscheint wenig aussichtsvoll. Die nur klinisch beobachteten Fälle können selbstverständlich nicht absolut beweiskräftig für die Behauptung eines Mischprozesses von multipler Sklerose und progressiver Paralyse sein. Als einwandfrei in der Entscheidung dieser Frage kommen nur die auch anatomisch nach den elektiven Methoden studierten Fälle in Betracht. Aber was das für Fälle waren, in denen man früher eine Mischung oder einen Utbergang von progressiver Paralyse und multipler Sklerose annahm, ist heute schwer zu entscheiden, da sie aus einer Zeit stammen, in der die anatomische Diagnose der Paralyse noch nicht genügend begründet und die Rindenveränderungen bei der echten multiplen Sklerose ebenfalls noch nicht genau studiert waren. Und eine anatomische Abgrenzung dieser Hirnerkrankungen von gewissen, großenteils auch heute noch unklaren, mehr oder weniger diffusen Prozessen konnte damals natürlich noch weniger sicher durchgeführt werden.

Im allgemeinen beweist wohl das Seltenerwerden der sog. t'bergangsformen zwischen multipler Sklerose und progressiver Paralyse in der Literatur, daß man bei dem früheren Stand der Kenntnis von beiden

1) Die Beziehungen zwischen der multiplen Sklerose und Dementia paralytica. Inaug.-Dissert. Berlin 1901.

2) Über psychische, insbesondere Intelligenzstörungen bei multipler Sklerose. Archiv f. Psychiatrie 40, 253. 
Erkrankungen viel $\mathrm{zu}$ oft einen kombinierten Prozeß annahm. In seinem Aufsatz über „Die psychischen Störungen bei der multiplen Sklerose" betont Raecke1) mit Recht, daß nicht immer genügend auseinander gehalten worden sei, ,ob zu einem typischen, paralytischen Prozeß sklerotische Herde sich zugesellt hatten oder ob durch übermäßige Ausbreitung einer disseminierten Hirnsklerose eine mehr diffuse Erkrankung des Cerebrums zustande gekommen war. Auch in letzterem Falle wird sich das klinische Krankheitsbild dem der Dementia paralytica annähern müssen."

Die Untersuchung schwerer und vor allem die Rinde betreffender Herdsklerosen lehrt die Richtigkeit dieses Satzes von $\mathrm{Raecke}{ }^{2}$ ). Er hat seine Geltung nicht nur für Fälle mit großen Mark- und Markrindenherden, sondern besonders für jene etwas selteneren Fälle, in welchen kleinste Herde in großer Anzahl die Rinde besetzt halten und in denen auch sonst feine, mehr diffuse Gliawucherungen im Markradius und in der obersten Rinde vorherrschen, - Fälle, die zweifellos echte multiple Sklerosen darstellen und die meines Erachtens noch ein aussichtsvolles Ziel für weitere anatomische Untersuchungen bieten dürften (vgl. die unten erwähnten Beobachtungen). Solche Fälle haben denn auch im klinischen Bilde viel Ähnlichkeit mit der progressiven Paralyse; sie heben sich durch die Intensität der psychischen Ausfallserscheinungen aus dem Gros der gewöhnlichen Fälle multipler Sklerose heraus, bei denen jedoch auch, wie wir besonders durch Raeckes und Seiffers Untersuchungen wissen, die psychischen Störungen weit häufiger sind, als man früher annahm. So erklärt es sich schließlich, daß noch heute in manchen Fällen die klinische Differentialdiagnose großen Schwierigkeiten begegnen kann bei der Entscheidung der Frage, ob Paralyse oder multiple Sklerose, obschon der klinische Symptomenkomplex, besonders das Verhalten der Pupillen, die No n nesche PhaseI-Reaktion und die Wassermannsche Reaktion im Blut und in der Cerebrospinalflüssigkeit doch in den meisten Fällen Klarheit bringen dürfte.

Das Vorkommen einer einfachen Kombination von multipler Sklerose und progressiver Paralyse kann zwar großes klinisch-diagnostisches und anatomisches Interesse besitzen, nicht aber von prinzipieller Bedeutung sein. Denn es ist, wie $\mathbf{M} \ddot{\text { üller }}{ }^{3}$ ) in seiner Monographie über die multiple Sklerose schreibt, bei der Häufigkeit dieser beiden Erkrankungen des Zentralnervensystems das gelegentliche Vorkommen von Kombinationen

1) Archiv f. Psychiatrie 41, H. 2.

2) Vgl. dazu außer der vorhin zitierten Arbeit R a ec kes auch seine neuere Publikation ,Zur forensischen Bedeutung der multiplen Sklerose“, Vierteljahrschr. f. gerichtl. Medizin. 1907.

3) Die multiple Sklerose des Gehirns und Rückenmarks. Jena 1904. S. 227. 
nicht unwahrscheinlich. Als Beispiel einer solchen Kombination kann u. a. wohl der Fall von $G a u^{1} p^{1}$ ) gelten, den er in seiner bekannten Arbeit über „Die spinalen Symptome der progressiven Paralyse“ kurz erwähnt hat. Ich verdanke Herrn Prof. Ga u p p einige kurze Notizen über diesen nicht ausführlicher publizierten Fall und durfte auch dank der Freundlichkeit des Direktors der städtischen Irrenanstalt in Breslau, des Herm Dr. Hahn, die Krankengeschichte dieses Falles einsehen. Der klinische Befund jedenfalls ließ an der Diagnose einer Paralyse keinen Zweifel; abgesehen von den psychischen Defektsymptomen (Merkfähigkeitsstörungen, Größenwahn, früher Abstumpfung des ästhetischen und ethischen Empfindens usw.) fanden sich als besonders wichtige neurologische Zeichen eine reflektorische Starre der Pupillen mit Miosis, ferner ausgesprochene Ataxie, Abschwächung und später Erlöschen der Patellarsehnenreflexe, Schlaffheit der Gelenke an den unteren Extremitäten, Sensibilitätsstörungen; später traten dazu spastische Symptome, wie besonders Dorsalklonus, Steifigkeit in den Beinen. Der Prozeß hatte sich mit ohnmachtsartigen Anwandlungen eingeleitet. Gegen Ende der Erkrankung zeigten sich bulbäre Symptome. Eine multiple Sklerose konnte nicht diagnostiziert werden, es wurde eine progressive Paralyse mit Tabes und bulbären und spastischen Symptomen angenommen.

Anatomisch entsprach den sensiblen Störungen eine mäßige aufsteigende Hinterstrangerkrankung. Die später auftretenden spastischen Erscheinungen erklärten sich aus multiplen Herden, die besonders im Halsmark und in der Medulla oblongata saßen. Es bandelte sich nach den Untersuchungen von Gaupp um typische sklerotische Herde. Dazu kam eine allgemeine Gehirnatrophie (ohne plaqueförmige Veränderungen), die allerdings, entsprechend dem damaligen Stande der Paralysepathologie (1895) nicht in jeder Beziehung einwandfrei als paralytisch analysiert werden konnte. Immerhin machten es das klinische .Bild, der allgemeine anatomische Befund im Großhirn und vor allem das eigenartige Nebeneinander von herdförmigen Rückenmarksveränderungen und selbständigen Hinterwurzeldegenerationen höchst wahrscheinlich, daß es sich da um eine Kombination von progressiver Paralyse mit multipler Sklerose gehandelt hat.

Abgesehen aber von einer solchen Koinzidenz zweier Krankheitsprozesse, von denen jeder nicht eben selten ist, sind wirkliche Utbergangsformen zwischen multipler Sklerose und progressiver Paralyse mit den Methoden der modernen neurohistologischen Analyse nicht sichergestellt. Auch hier ist es wohl in erster Linie wieder das elektive Zellbild Nißls gewesen, das einen klaren Einblick gestattete; und damit schwanden auch die ,Mischformen“.

1) Gaupp, Wernickes Arbeiten. 1898. 
Wie ungemein selten aber bei Paralyse sklerotische Herde im Rückenmark gefunden werden, geht auch daraus hervor, daß an dem großen, exakt untersuchten Material von $\mathrm{NiBl}$ und von $\mathrm{Alz}$ heimer Befunde, wie wir sie in unserem Falle erheben konnten, nicht zu verzeichnen waren, und daß F ürstner, der ja lange Jahre hindurch seine Studien über die Rückenmarksveränderungen bei der progressiven Paralyse fortgesetzt und darüber in vielen Vorträgen und Referaten berichtet hat, solche Fälle nicht erwähnt. Außer den systematischen Degenerationen in den Hinter- und Seitensträngen hat Fürstner auch den mehr diffusen und fleckigen Sklerosen der Rückenmarksstränge seine Aufmerksamkeit geschenkt; und er betont ausdrücklich, daß manche

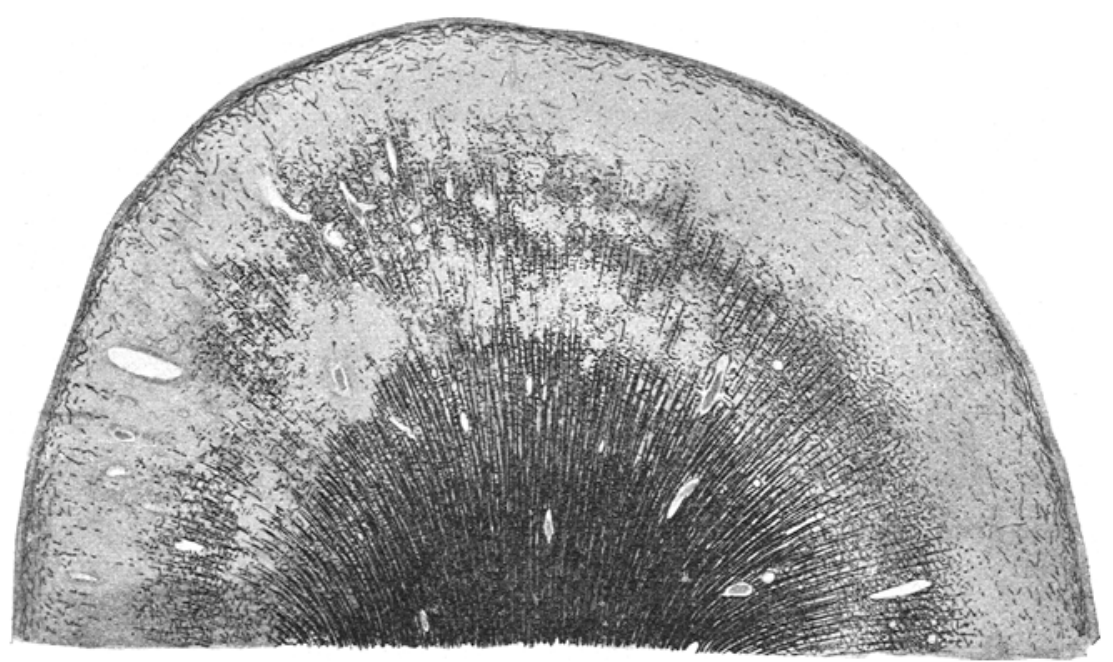

Fig. 4. Sehr unregelmäßige, kleine und größere marklose Flecken und Streifen. (Markscheidenfärbung am Gehirnsehnitt. Vordere Zentralwindung.)

plaqueförmige Gliawucherungen, die man neben der diffusen Vermehrung des Stützgerüstes $a b$ und zu in den Seitensträngen trifft, von den Herden der multiplen Sklerose getrennt werden müssen, wie das auch Gaupp tut. Es sind Gliaherdchen, in denen die nervöse Substanz, besonders auch die Achsenzylinder zerstört sind.

Ich selbst habe auch mindestens in 60 Fällen von Paralyse das Rückenmark mit den verschiedensten Methoden, besonders nach der Markscheidenfärbung, untersucht und nirgends ähnliche sklerotische Herde gefunden wie in dem Fall, von dem wir hier ausgingen.

Es wäre natürlich das einfachste, in diesem Falle lediglich eine zufällige Kombination einer progressiven Paralyse mit multipler Sklerose - ähnlich etwa der Ga u p pschen Beobachtung - anzunehmen. Merkwürdig ist dann freilich (und auch von dem Gauppschen Falle ab- 
weichend), daß es hier nur zur Bildung der drei Herde in der hintersten Partie der untersten Rückenmarksabschnitte gekommen ist; man müßte weiter auch annehmen, daß es sich um eine Art von anatomischer ,forme fruste " bzw. um einen ,,abortiven Typus" der multiplen Sklerose handelte, die keine Neigung zur Progression zeigte, da sie ja bei einem bereits 44 jährigen Manne lediglich zur Entwicklung der wenigen Herdchen geführt hatte. Ich weiß nicht, ob ähnliche Fälle von multipler Sklerose bekannt geworden sind, wo etwa vereinzelte Herde, die klinisch unentdeckt blieben, bei der anatomischen Untersuchung als „Nebenbefund" nachgewiesen werden konnten. Ich habe von derartigen Beobachtungen bisher nichts in Erfahrung bringen können.

Man wird wohl die Möglichkeit solcher abortiven anatomischen

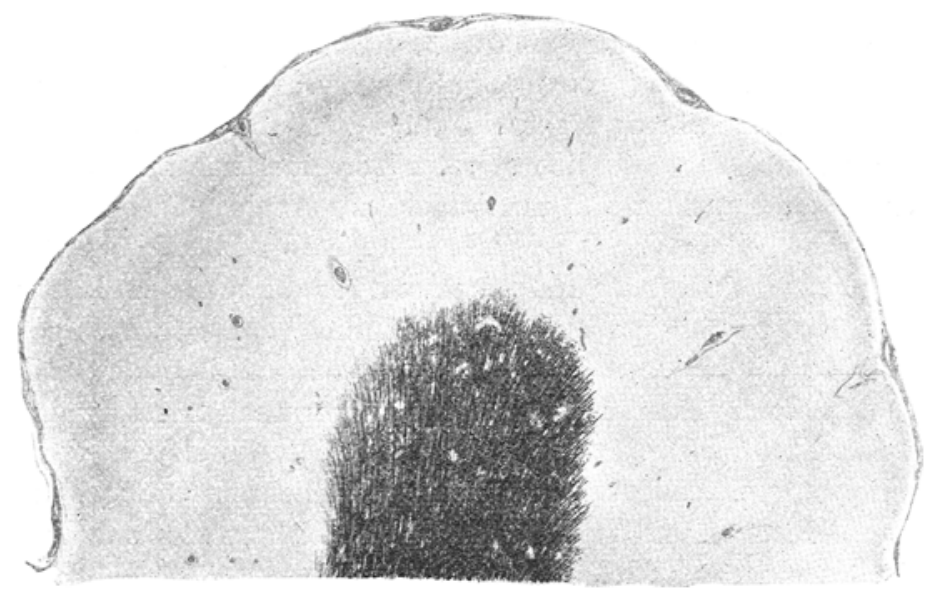

Fig. 5. Markleere Rinde. Die Ausstrahlung des Markradius wie abrasiert. (Kulschitzkypräp. I. Temporalwindung.)

Typen der multiplen Sklerose nicht von vornherein ablehnen und deshalb wohl auch in unserem Falle an die Kombination einer solchen Form mit einer progressiven Paralyse denken können. Aber es scheint uns doch, daß dieser herdförmigen Veränderung auch eine andere Deutung gegeben werden kann bei einem Vergleich mit den a uffallenden Befunden in der Hirnrinde, nämlich mit der Art des kortikalen Markfaserausfalls.

In fast allen Teilen des Großhirnmantels sehen wir neben diffusem Markfaserschwund herdförmig verteilte Ausfälle von Markfasern in den Aufsplitterungen des Markradius (vgl. die Abbildungen, Fall D.). Die fächerförmig ausstrahlenden Bündel erscheinen oft auf ziemlich scharf begrenzte Strecken wie abrasiert (Textfig. 5; Taf.VII, Fig. 2), anderwärts sind sie von marklosen Flecken unterbrochen, jenseits deren die Fasern wieder eine gute Markhülle zeigen, oder die Farbe erscheint von den 
Fasern eine Strecke weit wie weggewischt (Fig. 4). Manchmal greift auch der marklose Fleck bis in die Wurzel der Ausstrahlung nach dem kompakten Teil des Markradius über (Taf. VIII, Fig. 3 u. 4).

Wenn man diese Bilder mit den bekannten Beschreibungen des gewöhnlichen paralytischen Markschwundes, mit der Schilderung von dem Schwunde der Tangentialfasern, des supra- und intraradiären Flechtwerks und dem diffusen Untergang von Rindenmark vergleicht, dann müssen die Befunde hier zunächst als etwas im Paralysebild Fremdartiges imponieren. Nun zeigt aber nicht nur der klinische Verlauf dieges Falles, sondern auch vor allem der Rindenbefund am Zellpräparat nur die ty pischen Eigentümlichkeiten der Paralyse. Um Weitläufigkeiten zu vermeiden, genügt es wohl, wenn ich hervorhebe, daß die Rindenpräparate auch nicht etwa durch stärkere entzündliche progressive oder regressive Vorgänge an den Gefäßen, durch fleckige Rindenverödung oder Narben usw. von dem gewöhnlichen Rindenbild bei der Paralyse abweichen. Bietet so der Fall im Zell- und Gliabilde nichts Ungewöhnliches, so finden auch die zunächst fremdartig erscheinenden Markscheidenbilder ihre Analoga in den Befunden bei anderen Fällen von Paralyse; denn auch sonst begegnen wir bei Paralyse ähnlichen Bildernvon fleckigem Markschwund wie hier. Nur das ist etwas außergewöhnlich, daß der fleckige Markschwund in dem in Rede stehenden Fall nahezu in allen Teilen des Hemisphärenhirns mehr oder weniger ausgesprochen ist, während er sonst in den Fällen von Paralyse, in welchen er überhaupt vorkommt, gewöhnlich nur einzelne Windungen in auffälligerem Grade betrifft, der wesentlichste Grund wohl, weshalb ihm bislang so wenig Aufmerksamkeit geschenkt wurde.

Es liegt nun gewiß die Erwägung nahe: könnte es sich nicht bei den Skleroseherden im Rückenmark um im Prinzip ähnliche Bildungen handeln, wie bei den marklosen Flecken im Gehirn? Anstatt eine Kombination der paralytischen Hinterstrangerkrankung mit multipler Sklerose im Rückenmark anzunehmen, ließen sich da nicht jene vereinzelten Herde im Rückenmark doch als ein Produkt des paralytischen Prozesses selbst auffassen, wennschon es sich um einen recht seltenen Befund dabei handelt? Es mußte mit Rücksicht darauf nach Anhaltspunkten gesucht werden, die etwa die Vermutung stützen könnten, daß wir es bei den Rückenmarks- und den Rindenherden mit analogen pathologischen Produkten zu tun haben.

Der Versuch einer Lösung dieser Frage hat zunächst von der Erörterung über Art und Verteilung des herdförmigen Markschwundes bei der Paralyse überhaupt auszugehen; und ich werde deshalb hier ganz allgemein mitteilen, was ich über Häufigkeit, Gestalt, 
Form usw. der markarmen Rindenflecke bei Paralyse feststellen konnte, nachdem ich ein paar Worte über die in dieser Frage interessierende Literatur vorausgeschickt habe.

Uber das Vorkommen von Herden bei der Paralyse ist nicht selten berichtet worden, so z. B. von Siemerling gelegentlich seines Vortrags über Technik und Härtung großer Hirnschnitte ${ }^{1}$ ). Auch in solchen Fällen, in denen klinisch Herderscheinungen nicht bestanden, konnte Siemerling zahlreiche sklerotische Flecke in der Rinde, dem Mark und den großen Ganglien feststellen. An manchen Stellen der Rinde waren sie außerordentlich zahlreich; die kleinen sklerotischen Herde fanden sich immer in der Umgebung eines Gefäßes und die Gefäße waren deutlich erkrankt; häufig zeigten letztere eine ausgesprochene Verdickung ihrer Wandung. Auch im Balken und im Mark des Hinterhauptlappens fanden sich in einem Falle multiple sklerotische Herde; in der Insel waren Gefäßlücken wahrzunehmen. ťber den Zusammenhang der Herde mit dem paralytischen Prozeß ließ sich nichts ermitteln. Von einer sekundären Degeneration erwiesen sich die Herde unabhängig, dagegen fielen sie durch ihre Beziehungen zu erkrankten Gefäßen auf. In der Diskussion zu diesem Vortrag berichtete Vogt von weitverbreiteten sklerotischen Herden in der Umgebung verdickter Gefäße bei einem Fall von progressiver Paralyse. Es handelte sich dabei nicht um sekundäre, sondern um primäre sklerotische Prozesse. Während in den Siemerlingschen Beobachtungen den anatomisch nachweisbaren Herden klinisch keine Lokalerscheinungen entsprachen, fand Heilbronner ${ }^{2}$ ) zu klinischen Symptomen (motorischen Ausfallserscheinungen nach Anfällen) in der betreffenden Region akute Zellveränderungen in fleckiger Anordnung, wie eine solche auch für den Ausfall der Fasern an zahlreichen Präparaten nachzuweisen sei. Schaffer ${ }^{3}$ ) berichtet in seinen „Vorträgen" von kleinen perivasculären Inseln in den Zentralwindungen, und zwar in der Höhe der Markstrahlen bzw. der großen Pyramiden. Sie bewirken nach seinen Untersuchungen eine absteigende Degeneration. In diesem Zusammenhang weist Schaffer auch auf die Beschreibung hin, welche Binswanger von dem inselförmigen, fleckartigen Charakter des Faserschwundes an manchen Paralysepräparaten gibt. Schließlich haben sich, wie wir eingangs hervorhoben, $B \circ \operatorname{rda}^{4}$ ) und später

1) Siemerling, Über Technik und Härtung großer Gehirnschnitte. Vortrag. Allgem. Zeitschrift f. Psychiatrie 56.

2) Heilbronner, Rindenbefunde bei progressiver Paralyse. Vortrag. Allgem. Zeitschrift f. Psychiatrie 53.

3) Schaffer, Anatomisch-klinische Vorträge aus dem Gebiete der Nervenpathologie. Jena 1901. S. 244.

4) Borda, Paralysie générale progr. Rev. de la soc. méd. argent. Tome XIIr. 1906. (Zitiert nach Fischer.) 
Fischer ${ }^{1}$ ) um die Schilderung eines fleckigen Schwundes der corticalen Markfaserung verdient gemacht. Bord a beschreibt auch (nach Fischers Zitat) ähnliche Herde, wie in der Rinde, im Hirnstamm. Beide heben die Beziehungen der Herde zu Gefäßen hervor, welche jedoch keine besondere Wandveränderung - im Gegensatz zu den Befunden Siemerlings, Vogts u. a. - zeigen, und ferner betonen sie das Fehlen einer sekundären Degeneration im Anschluß an die Herde, wie eines erheblichen Achsenzylinderausfalls in denselben.

Man sieht, daß in diesen Mitteilungen über Herde bei Paralyse offenbar histologisch und pathogenetisch verschiedenartige Veränderungen gemeint sind. Es dürften an dem Zustandekommen von Herden verschiedene Prozesse schuld sein, die zum Teil auch mit der paralytischen Erkrankung selbst direkt nichts zu tun haben, wie gröbere regressive Gefäßveränderungen, entzündliche Vorgänge syphilitischer Art usw. Wir können die Frage hier nicht erörtern, welche verschiedenartigen herdförmig lokalisierten Prozessen jenen Befunden zugrunde lagen. Wir wollen uns hier nur mit dem fleckigen Markschwund in der Rinde beschäftigen.

Von diesem geben die beigefügten Figuren einiges wieder. In dem Fall D., von welchem wir ausgingen, sind ja fast alle Formen und Verteilungsarten der markarmen Herde zu finden. Je mehr man sich mit solchen Markfaserbildern beschäftigt, desto mehr findet man, was der Illustration wert erscheint; aber auch das hier Gegebene dürfte wohl genügen, um wenigstens von den häufigsten Bildern eine Vorstellung zu ermöglichen. Sehr oft begegnet man kleinen Flecken, die ziemlich dicht beieinanderstehend, das Intraradiärgebiet einnehmen und der ganzen Rinde oft ein gesprenkeltes Aussehen verleihen (Taf. VII, Fig. 1). Die Herdchen durchsetzen in Reihen den oberen Ausstrahlungsbezirk der Radiärfasern oder sie sind auch dicht darunter in der Höhe etwa des Gennarischen Streifens gelegen, so daß über ihnen noch eine markhaltige Zone von ausstrahlenden Markbündeln und transversal ziehenden Geflechten bleibt. Dann gibt es größere und kleinere vereinzelte Herde, bald mehr am Ursprung des Markfächers, bald höher oben in der Rinde. Manche Herde sind völlig markleer und haben häufig ganz scharfe Grenzen; bei anderen handelt es sich mehr um eine Lichitung der Markfaserung, die wenigstens zum Teil auf einem bloßen Dünnerwerden der Markscheide der Faser beruht. Das Abgerundete der Herde tritt in anderen Bildern nicht so deutlich in die Erscheinung. Man sieht vielmehr auf größere oder kleinere Flächen oder in Bänder- und Streifenform die Markfärbung ausgelöscht (vgl. Textfig. 4; Taf. IX, Fig.6). Mitten

1) Fischer, Utber den fleckweisen Markfaserschwund in der Hirnrinde bei progressiver Paralyse. Arbeiten aus der deutschen psychiatrischen Universitätsklinik in Prag 1908. 
in einer sonst markreichen Rinde können Teile des Markfächers eine Strecke weit unterbrochen sein. Sehr merkwürdig und gar nicht selten sind dann die Rinden, in welchen die Markbündel hart an ihrer Wurzel wie abgebrochen oder abgefressen erscheinen, es sieht wie ,Mottenfraß" aus. Buchten- und bogenförmig oder auf ganze Strecken hin kann so die Rindenfaserung, die in den unmittelbaren Nachbarbezirken derselben Windung noch kaum reduziert ist, gleichsam abrasiert erscheinen und die Rinde dort vollständig markleer sein. So kommt es zu den zunächst verblüffenden Bildern gänzlich markloser Rinden, die keine Volumensabnahme zeigen, in denen im Gegenteil das Rindenband durch die Reduktion des Markes besonders breit erscheint (Fig. 5). In anderen Fällen sieht man deutlich, wie der fleckig beginnende Markschwund zu einer Rindenverödung führt; Reste der Markfaserung zeigen schließlich noch die ursprünglichen Herdgrenzen an.

Viel seltener, als die eben geschilderte Art und Verteilung des fleckigen Markschwundes, ist das Ưbergreifen eines Herdes auf die Wurzeln des Markfächers. An solchen Präparaten ist der Herd besonders scharf aus der gesunden Umgebung herausgeschnitten (Taf. X, Fig. 7), auch wenn er, wie in Taf. VIII, Fig. 4 noch nicht zur völligen Entmarkung der betreffenden Zone geführt hat. Auch bei diesen Herden bleibt die untere Grenze noch ganz dicht unter der Wurzel der ausstrahlenden Markbündel, also noch $\mathbf{m}$ der Gegend der tiefen Rinde in der untersten Ganglienzellschicht; daß er darüber hinaus auch in die eigentliche weiße Substanz hineingreift, ist äußerst selten (Taf. XI u. XII, Fig. 9-11). Auf die Bedeutung dieses Befundes komme ich später noch zu sprechen.

Von einer weiteren Beschreibung des fleckigen Markschwundes auf Grund des Markscheidenpräparates kann ich wohl absehen. Ich habe nur noch zu erörtern, wie es mit der Verteilung dieser Herde über die verschiedenen Rindengebiete und mit der Häufigkeit ihres Vorkommens überhaupt steht. Daß der fleckige Markschwund bei der Paralyse lange nicht so häufig und regelmäßig gefunden wird, wie der einfache mehr oder weniger gleichmäßige Markausfall, das kann man ja schon aus der Tatsache entnehmen, daß er, außer in den Arbeiten Fischers und Bordas, in der Literatur nicht die ihm zukommende Würdigung gefunden hat. Zum Teil mag das auch daran liegen, daß häufig nur einige Präparate nach der Markscheidenmethode angefertigt werden und daß wohl auch die Technik nicht immer völlig ausreicht, Aber das ist sicher: je mehr man nach solchen Herden sucht, desto regelmäßiger findet man sie bei der Paralyse und desto seltener werden die anfangs so sehr viel häufigeren Fälle, die nur einen gleichmäßigen Ausfall aufzuweisen schienen. Auch aus Fi schers Mitteilung läßt sich das schließen. Der Prozentsatz seiner Fälle mit fleckigem Markscheidenschwund ist, 
gegenüber seiner ersten Mitteilung, jetzt auf $65 \%$ angestiegen ${ }^{1}$ ); und ich selbst war überrascht bei der Durchuntersuchung des mir gerade zur Verfügung stehenden und für diese Publikation verarbeiteten Materials in 13 von 25 Fällen eine ausges prochene fleckige Markatrophie in der Rinde zu finden. Ubrigens wurden bei diesen Untersuchungen von jedem Gehirn mindestens aus 12 verschiedenen Windungen Markscheidenpräparate angefertigt.

Nur 12 Fälle boten, soviel wir sie durchforschten, nirgends eine Andeutung von fleckigem Ausfall. Es waren darunter zwei ganz beginnende Fälle, übrigens auch der, von dem ich jüngst in anderem $\mathrm{Zu}$ sammenhange berichtet habe $\left.{ }^{2}\right)$. Aber auch Fälle von Paralyse mit ausgedehnten entzündlichen Veränderungen und starkem Markfaserschwund und solche mit erheblicher Atrophie gehören dazu. Von den 13 Fällen mit fleckiger, corticaler Markatrophie sind es 6, die nur an einzelnen Windungen Herde aufweisen, manche in der Art, daß sie in dem beschränkten Rindéngebiet, wo sie überhaupt vorkommen, nur klein und spärlich sind, und andere wieder so, daß der betreffende Bezirk auffallend reich von Herden durchsetzt ist und das Bild des corticalen „Markfraßes" sehr ausgeprägt erscheint, während die Rinde sonst nur gleichmäßig an Markfasern reduziert ist. Daran reihen sich zwei Fälle mit etwas weiterer Verbreitung des fleckigen Markschwundes, sie leiten hinüber zu den 5 Fällen mit starker, herdförmig verteilter Markfaseratrophie in den verschiedensten Rindengebieten; natürlich ist die Trennung zwischen diesen Gruppen mit geringem oder mäßigem und mit starkem herdförmigen Markscheidenausfall nicht scharf durchzuführen. Ich sondere die letzten 5 Fälle nur deshalb von den anderen ab, weil sie eben in ganz besonders ausgesprochenem Maße die fleckigen Degenerationen zeigen.

Ich kann nach meinem Material noch nicht entscheiden, welche Windungen am häufigsten betroffen sind. In den Fällen mit geringen Graden des fleckigen Markscheidenschwundes ist dessen Hauptsitz bald die hintere Zentralwindung und der Parietallappen, bald die Stirnregion; und in den Fällen mit sehr starker Ausprägung jener eigentümlichen Rindenveränderung ist es bald mehr das Stirnhirn, bald auch die Inselgegend und die Zentralwindungen oder der Temporallappen, welche am meisten betroffen erscheinen; auch der Hinterhauptslappen ist in den extremen Fällen in der Regel deutlich in jener charakteristischen Weise erkrankt. Im allgemeinen kann ich auf Grund dieser nicht sehr umfangreichen Untersuchung nur sagen, daß auch diese merkwürdige

1) Fischer, Die Lues-Paralyse-Frage. Referat. Allgem. Zeitschrift für Psychiatrie 66. 1909.

2) Zur Frage vom Wesen der paralytischen Hirnerkrankung. Zeitschrift für die gesamte Neurologie u. Psychiatrie Orig. 1, 105. 1910. 
Teilerscheinung der paralytischen Rindenerkrankung am ausgesprochensten und häufigsten in den vorderen $z$ wei Dritteln des Hemisphärenhirns ist, speziell in der Zentralregion, den oberen Temporalwindungen und dem Fuße der ersten und zweiten Frontalwindung. Natürlich ist über diese Frage der Verteilung der Herde nur auf Grund eines wesentlich größeren Materials und einer Vergleichung der Untersuchungsergebnisse verschiedener Autoren Klarheit zu bringen.

Es ergibt sich somit aus dieser Utbersicht über das Vorkommen von fleckweise verteiltem Markschwund in der paralytischen Rinde, daß es sich dabei um einen gewiß nicht seltenen Befund handelt. Ich stimme Fischer darin durchaus zu, und ich halte den Widerspruch, den Fischers Mitteilungen gefunden haben, für unberechtigt. Es wird ihm besonders der Einwand gemacht, daß auch bei anderen Rindenerkrankungen die gleichen fleckigen Marklichtungen zu finden sind, wie sie Fischer für charakteristisch bei Paralyse hält, nämlich z. B. bei Arteriosklerose und anderen Ernährungs- und Zirkulationsstörungen in der Rinde. Von einer Identität der Flecke dort mit denen bei der Paralyse kann aber keine Rede sein. Das lehrt ja schon der Befund am Weigert - Präparat, der nur an die Bilder bei multipler Sklerose erinnert, und das lehrt weiter vor allem die histologische Analyse der Marklichtungen. Mit dieser wollen wir uns nun beschäftigen und dabei vor allem die Ähnlichkeit der paral y tischen Rindenherde mit den Plaques bei der multiplen Sklerose berücksichtigen.

Für die genauere histologische Analyse der Rindenbezirke, in welchen das Markscheidenpräparat einen fleckigen Markfaserschwund anzeigt, und zum Vergleich der histologischen Detailbefunde hier mit denen bei multipler Sklerose kam es darauf an, unmittelbar aufeinanderfolgende, nach den verschiedenen Methoden behandelte Schnitte nebeneinanderstellen zu können. Es ist ja für eine solche Vergleichung die größte Schwierigkeit, daß die verschiedenen Färbeverfahren eine verschiedene Vorbehandlung des Materials brauchen, daß speziell die Markscheidenfärbung von der Rinde nur an chromierten eingebetteten Blöcken wirklich zureichende Bilder gibt, daß dann aber befriedigende Achsenzylinder- und Gliapräparate nicht herzustellen sind usf.

Es wurde dazu notwendig, nach einem Verfahren zu suchen, das am Gefrierschnitt von Formolmaterial eine gute Markscheidenfärbung liefert, damit von dem gleichen Block auch Achsenzylinder-, Glia-, Zell- und Fettpräparate zum Vergleiche gewonnen werden können. $\mathrm{Da}$ die bisher unternommenen Versuche die Markscheide der Rinde am Gefrierschnitt zur Darstellung zu bringen, nicht genügen, ist oft betont worden; ich habe bei der Veröffentlichung meiner neuen Methode kurz darauf hingewiesen. Es gelang nun eine sehr einfache sichere Methode ausfindig zu machen, über die ich vor kurzem im ,Neurologi- 
schen Centralblatt" 1 ) berichtet habe. Bei diesem Verfahren ist es von nebensächlichem Interesse, daß man schon rasch (wenige Tage nach der Sektion) ein gutes Markscheidenbild erlangen kann $^{2}$ ); das Wichtige, was sie bringt, ist eine sehr vollständige Darstellung der Mark fasern der Rinde (und natürlich auch sonst am Nervensystem), die wir bequem mit den Achsenzylinder-, Zell-, Fettpräparaten und den Gliabildern vergleichen können.

Ich wende das Verfahren jetzt in folgender Weise an: Gefrierschnitte von formolfixiertem Material kommen für 6-10 Stunden in eine 21/2 prozentige Lösung von schwefelsaurem Eisenammoniumoxyd, dann werden sie für einige Minuten in Wasser abgespült, für 15 Minuten in 70 prozentigen Alkohol und von da in eine alte Hämatoxylinlösung übertragen (5 Teile einer 10 prozentigen alkoholischen Hämatoxylinlösung auf 100 Teile Wasser). Dort bleiben sie etwa 12 Stunden in gedeckten Schalen stehen. Nach Abspülen in Wasser werden sie in der Beize von schwefelsaurem Eisenammoniumoxyd differenziert. Am besten wechselt man die Differenzierungsflüssigkeit einmal, dann längeres Auswaschen (ca. 1-2 Stunden), Entwässern, Xylol, Balsam.

Die Schnitte haben einen ähnlichen Ton wie Pal-oder Kulschitzky. Wolters-Präparate. Bezüglich der Vollständigkeit der Färbung auch der feinsten Rindenfasern, über welche ich mich bei der Niederschrift jener Mitteilung über meine Methode (Jan. d. J.) sehr zurückhaltend äußern mußte, da ich erst verhältnismäßig geringe Erfahrungen hatte, darf ich mich heute, nachdem ich die Markscheidenmethode am Gefrierschnitt inzwischen sehr viel und an dem verschiedensten Material angewendet habe, wohl etwas bestimmter aussprechen: soviel ich sehe, gibt das Verfahren eine sehr vollständige Färbung gerade auch der feineren Markgeflechte der obere n Rinde, und es ist nicht selten der Kulschitzk yschen Methode, welche wohl sonst am chromierten und eingebetteten Material die besten Resultate liefert, in der Darstellung des Faserreichtums der Rinde überlegen; ich habe das auch an Material, das sich nach den sonst üblichen Markscheidenmethoden nicht recht färben will, mehrfach beobachtet. Ich glaube versichern zu können, $d a ß$ das nicht etwa an einer mangelhaften Beherrschung jener Methoden liegt.

Zur Technik möchte ich noch bemerken, daß das Wiederholen von Färbung und Differenzierung, auf das ich anfangs und in meiner Mitteilung besonderen Wert legte, in letzter Zeit nur ausnahmsweise notwendig wurde. Das liegt wohl daran, daß mit der häufigeren Benutzung der Hämatoxylinlösung zu diesem Verfahren ihre Färbekraft stark zugenommen hat. Es genügt jetzt, die Schnitte 12 Stunden hintereinander (selten länger) in der Farbe zu lassen und danach nur einmal zu differenzieren. Von einer Wiederholung der Färbungs- und Differenzierungsprozedur kann ich jetzt, wie gesagt, fast immer absehen. Utbrigens ist dann auch der Ton der Präparate häufig viel schöner und heller, während er sonst oft schmutzig gelbgrau wurde. Rückenmarks-, Hirnstamm- und Kleinhirnschnitte brauchen in der Regel nur 3-4 Stunden in der Farbe zu liegen.

Das Gelingen der Färbung hängt - das möchte ich hier noch ausdrücklicher als in meiner ersten Mitteilung hervorheben - vorwiegend davon

1) Markscheidenfärbung am Gefrierschnitt. Neurol. Centralbl. 1910.

2) Mit dieser Methode kommen übrigens auch gewisse strukturelle Eigentümlichkeiten der Rückenmarkswurzeln und Nerven brillant zur Darstellung. Herr Dr. Essick vom anatomischen Institut in Baltimore wird darüber demnächst berichten. 
$a b$, daß man eine für die gleiche Methode bzw. für die Heidenhainsche Kernfärbung schon sehr oft benutzte Hämatoxylinlösung verwendet, die man nach dem Gebrauch immer wieder durch das gleiche Filter zurückgießt. An dem Alter der Hämatoxylinlösung allein liegt der Erfolg der Färbung nicht, sondern die Lösung erhält erst durch die längere Benutzung, besonders wohl durch die allmähliche Zumischung des den Schnitten anhaftenden Eisenalauns, ihre Färbekraft.

Ein besonderer Vorteil der Methode scheint mir auch darin zu liegen, daß man an den Schnitten, wenn sie bereits gefärbt und differenziert sind, noch die Weigert sche Gliafärbung anwenden kann und daß dieser Versuch, wenigstens am pathologischen Präparat, oft ganz befriedigende Resultate gibt. Man braucht nur die Schnitte in der Beize stark auszudifferenzieren, danach zu wässern und dann in das übermangansaure Kali zu tun. Es folgt nach den Weigertschen Vorschriften die Entfärbung und Reduktion in Chromogen-Ameisensäure; dann kommt der Schnitt ins Wasser und für einige Zeit in 70 prozentigen Spiritus. Von da bringe ich die Schnitte auf den Objektträger, auf welchem ich eine Spur von EiweiBglycerin zum besseren Haften der Schnitte verrieben habe; nach Übergießen mit Ätheralkohol trocknet, beim Verdunsten desselben, der Schnitt am Objektträger fest und kommt dann in die Farbe. Ich benutze mit wesentlichem Vorteil vor der gewöhnlichen gesättigten alkoholischen Methylviolett- eine Karbol Methylviolettlösung in der Weise, wie ich es in dem Anhang zu der KahldenGierkeschen Technik beschrieben habe. (8. Aufl. 1909.) Differenzieren usw. wie gewöhnlich. Mehrfach fand ich übrigens, daß fertige Gliapräparate, die nur unvollkommen gefärbt waren, bei einer späteren Wiederfärbung, nach Ablösung des Canadabalsams und Entfärbung in Alkohol, eine sehr viel vollkommenere Darstellung der Gliafasern zeigten; worauf dieses merkwürdige Verhalten beruht, konnte ich nicht herausfinden. Daß es aber gar nicht selten beobachtet werden kann, ist sicher.

Selbstverständlich gelingt die „Markscheidenfärbung am Gefrierschnitt" auch an solchem Material, das sogleich in Gliabeize (+ Formolzusatz) eingelegt war. Ich erwähne das im Hinblick auf die jüngsten Mitteilungen von AIzheimer'), wonach es für eine gute Darstellung der Gliazelleiber usw. nach den von ihm angegebenen Methoden notwendig ist, die Stücke von vornherein in der Fluorchrombeize zu fixieren. Man kann somit auch diese Alzheimersihen Gliazellbilder mit den Markscheidenpräparaten vergleichen.

Untersuchen wir mit der neuen ,Markscheidenfärbung am Gefrierschnitt" die paralytische Rinde und vergleichen wir die Bilder von den marklosen Flecken dort mit den Befunden, die das Bielschowskysche Achsenzylinderpräparat gibt, so imponiert von vornherein als wichtigste histologische Eigentümlichkeit die von Borda und Fischer bereits betonte Persistenz der Achsenzylinder in den Herden und marklosen Bezirken. Schon das Markscheidenpräparat macht es ja, wie ich eingangs hervorhob, sehr wahrscheinlich, daß die Nervenfasern in den abgegrenzten Herden und Lichtungen nicht völlig zugrunde gegangen sind. Man sieht, wie die Markfasern plötzlich in ihrem Verlauf unterbrochen werden und oft jenseits des

1) Alzheimer, Beiträge zur Kenntnis der pathologischen Neuroglia und ihrer Beziehungen zu den Abbauvorgängen im Nervengewebe. Histologische und histopathologische Arbeiten über die Großhirnrinde. III. 3.

Z. f. d. g. Neur. u. Psych. O. I. 
Herdes wieder auftauchen. Wie abgehackt oder abgefressen erscheint die Ausstrahlung des Markradius über mehr oder weniger große Bezirke einer Windung. Manche Fasern setzen sich in den Lichtungen mit stark verdünnter Markhülle eine Strecke weit fort. Ein feiner grauer Streifen - die Fortsetzung der jenseits des Herdes intensiv geschwärzten kräftigen Markhülle - umgibt solche Fasern im Herd. Von den Herd- und Streifenbildungen am Markscheidenpräparat, von dem plötzlichen Abgeschnittensein des in die Rinde ausstrahlenden Markfächers merkt man an dem Bielschowsky - Präparat nichts. Ich glaube darauf verzichten zu können, an Abbildungen diese Tatsache zu demonstrieren. Man kann sich ja davon unschwer überzeugen, wenn man das Markscheidenbild vom Gefrierschnitt mit dem vom gleichen Block gewonnenen Bielschowsk y - Präparat vergleicht. Besonders einfach liegen die Verhältnisse für diese Vergleichung natürlich in den Rinden, die stellenweise völlig entmarkt sind und in denen der Radius seiner fächerförmigen Ausstrahlung schon am Ursprunge beraubt ist, wie z. B. an Schnitten, die den in Textfig. 5 u. Taf. IX, Fig. 5 abgebildeten Windungen entsprechen; man sieht da die Fasern des Radius in derben Bündeln in die Rinde eindringen, und in der Regel kann man auch am Farbton im Bielschow sky-Präparat erkennen, wo die Nervenfaser ihr Mark verliert und marklos weiterzieht.

Natürlich tritt an der stark erkrankten paralytischen Rinde auch am Bielschowskyschen Achsenzylinderpräparat eine Reduktion des Fasergehaltes deutlich zutage. (Vgl. auch Bielschowsky und Brod$\left.\operatorname{man} n^{1}\right)$ u. a.) Was aber hier wichtig erscheint, ist eben die Tatsache, daß die Herde, Flecken und Lichtungen des Markbildes sich am Achsenzylinderpräparat nicht etwa infolge eines Fasermangels abheben, daß sie dort vielmehr überhaupt nicht als Plaques hervortreten, und daß der im Markscheidenbild oft so scharfen Unterbrechung der derben Radiärbündel keineswegs eine Zerstörung der betreffenden Achsenzylinder entspricht.

Weiterhin ist von Wichtigkeit, daß auch a $m$ Zell präparate und i $m$ Neurogliabilde die Stellen der Herde und Lichtungen sich nicht durch besondere histologische Eigentümlichkeiten vor ihrer Umgebung a uszeichnen, daß der fleckige Markfaserschwund nicht etwa, wie man erwarten könnte, darin sein Spiegelbild findet. Der genaue Vergleich zwischen dem Markscheidenbilde und dem ebenfalls vom Gefrierschnitt gewonnenen, korrespondierenden Toluidinblaupräparate zeigt in den den Flecken entsprechenden Stellen nichts Auffallendes und Herdförmiges. Ein solches am Gefrierschnitt hergestelltes

1) Bielschowsky und Brodmann, Zur feineren Histologie und Histopathologie der Großhirnrinde mit besonderer Berücksichtigung der progressiven Paralyse. Journal f. Psych. u. Neurol. 1905. 
Zellpräparat kann zwar kein Äquivalentbild im Sinne Nißls geben, aber es erlaubt doch wenigstens eine Orientierung über die Menge der Ganglien- und Gliazellen und über etwaige gröberer Untergangs- bzw. Wucherungserscheinungen. Was wir da von Abnormitäten feststellen können, unterscheidet sich von den Veränderungen der Umgebung nicht; die Intensität der Ganglienzellenerkrankung und -auflösung bzw. der Gliazellvermehrung ist in den fleckigen Lichtungen nicht größer als anderwärts. Und auch am Gliafaserpräparat heben sich die mark losen Flecken nich t etwa als Bezirke mit kompakter oder dichter Gliafaserwucherung ab. An Rinden, an denen die Gliafaserfärbung am Gefrierschnitt (s. o.) durchaus brauchbare Resultate gab, war davon nichts wahrzunehmen; nur manchmal hatte man den ,Eindruck“, daß die allenthalben sich findenden großen und kleinen Spinnenzellen mit gebündelten Fortsätzen in der Gegend der Herde etwas reichlicher seien. Dort, wo es zu einer tief nach dem Radius zu herabreichenden und scharf begrenzten Entmarkung der Rinde gekommen ist, kann man sich jedoch häufiger von einer lokal stärkeren Wucherung faserproduzierender Spinnenzellen in der tiefen Rinde überzeugen, z. B. an solchen Windungen, von denen Taf. IX Fig. 5 eine Illustration gibt.

Auch die Gefäßveränderungen in den Herden und überhaupt an den Rinden mit starkem, fleckigem Markschwund zeigen weder qualitativ noch quantitativ etwas Besonderes. Die entzündlichen und die regressiven Erscheinungen an den Gefäßwänden sind dort nicht etwa stärker ausgesprochen. Das betonen auch Borda und Fischer, die im übrigen - besonders Fischer enge Beziehungen zwischen Herden und Gefäßen annehmen. Ich habe mich nicht sicher davon übèrzeugen können, daß ein inniger Zusammenhang regelmäßig zwischen Herden und Gefäßen besteht, obschon auch ich Bilder gesehen habe, die, wie Taf. VII Fig. 1 sehr für einen solchen zu sprechen scheinen. Aber besonders für die streifenförmigen und breiten Lichtungen und für den ,Markfraß $\beta^{\prime \prime}$ an der Wurzel der Radiärfasern habe ich geographische Beziehungen zwischen Herden und Gefäßen nicht feststellen können. Und bei den kleinen Plaques ist deren Lage zu einem Gefäß so ungemein wechselnd, insofern nämlich das Gefäß bald im Mittelpunkt, bald aber ganz in der Peripherie oder doch stark exzentrisch liegt, daß ich pathogenetische Beziehungen zwischen fleckigen Marklichtungen und Gefäßen nicht zu erkennen und mir vorzustellen vermag. Die Häufigkeit, mit der im Bereiche der Herde, selbst der kleinen, ein Gefäß gefunden wird, muß ich mir doch, trotz Fischers Einwand, vor allem aus dem pathologischen Gefäßreichtum der paralytischen Rinde erklären. Mit Rücksicht auf die Gestalt der Herde und ihre wechselnde Lage zu den Gefäßen ist 
mir die pathogenetische Bedeutung der Zirkulation oder gar einer ganz hypothetischen, fleckweise wirkenden Noxe nicht verständlich.

Das wichtigste Ergebnis dieser histologischen Analyse der marklosen Flecken ist also: daß dem fleckigen Markscheidenschw und herdförmige Veränderungen an dem funktionstragenden Nervengewebe sonst und an der Glia und den Gefäßen nicht entsprechen.

Dieses Ergebnis ist wenig befriedigend. Denn man möchte doch das, was in den marklosen Herden an die Stelle des untergegangenen Markes getreten ist, färberisch zur Darstellung bringen und ein Positiv zum Markscheidenbild finden. Ich habe mich viel darum bemüht, aber es ist mir bei diesen Rindenherden der Paralyse nicht gelungen. Auch mit den neuen Alzheimerschen Methoden, die uns ja jetzt bereits über vieles, was vordem verborgen war, Aufschluß geben, kam ich nicht recht weiter. Es sind freilich nur 5 von meinen Paralysen, die ich nach den neuen Methoden von Alzheimer untersuchen konnte, darunter aber 2 mit sehr ausgedehnter Herdbildung und mit ausgesprochenem „,corticalen Markfraß“. Man sieht bei der Alzheimerschen Hämatoxylin- (Mallory-) Färbung (am Gefrierschnitt des in Gliabeize fixierten Blocks), wie zahlreiche faserbildende und einfachzellige, auch amöboide Gliaelemente die Bezirke der Marklichtung bevölkern, aber diese Partien fallen doch nicht etwa durch eine erhebliche Vermehrung ihrer Gliazellen als Herde gegenüber ihrer Umgebung auf. Bei der tief in die Wurzel der Radiärfaserung herabreichenden Markscheidenzerstörung ist jedoch die Wucherung der Gliazellen auch lokal deutlich. Das Fettpräparat gibt keinen besonderen Aufschluß.

In diesem negativen histologischen Verhalten der marklosen Herde bei anderen Elektivfärbungen berührt sich der corticale fleckige Markschwund bei der progressiven Paralyse auf das engste mit den Plaques bei der multiplen Sklerose. Denn auch bei den lediglich in der Rinde gelegenen Skleroseplaques fehlen alle anatomischen Herdveränderungen im Achsenzylinder-, Zell- und Gliafaserpräparat.

Von dem Fehlen herdförmiger Veränderungen im Zell- und Achsenzylinderbild bei den Rindenplaques ist mehrfach berichtet worden; ich brauche mich hier nicht in Erörterungen darüber einzulassen. Merkwürdig wenig bekannt dagegen ist, daß auch am Gliafaserpräparat keine herdförmigen Gliawucherungen an den Stellen der corticalen Skleroseherde gefunden werden.

Ich verweise in diesem Zusammenhang auf die Arbeit von Gustav Oppenheim ${ }^{1}$ ), der sich in eingehenden Untersuchungen mit den

1) Gustav Oppenheim, Zur pathologischen Anatomie der multiplen Sklerose mit besonderer Berücksichtigung der Hirnrindenherde. Neurol. Centralbl. 1908. 
Rindenherden der multiplen Sklerose beschäftigt hat. Er konnte zeigen, daß in den corticalen Plaques der multiplen Sklerose eine kompakte Gliafaserwucherung fehlt. „Kleinere Herde, die vollständig in den Meynertschen Schichten der Großhirnrinde gelegen waren und hier durch die Markscheidenfärbung in deutlich zirkumskripter Form dargestellt wurden, weisen in ihrem Areal nur zerstreute, gebündelte Gliazellen auf, so da $\beta$ man auf Grund des Gliabildes allein gar nicht darauf gekommen wäre, hier einen Herd zu vermuten." Es zeigte sich ferner, „daß an Stellen, wo makroskopisch ein Herd in ovaler, rundlicher oder unregelmäßiger Form von der Marksubstanz auf die Rinde übergreift, und wo die Markscheidenmethode das Negativbild des Herdes in dieser Ausdehnung darstellte, daß an solchen Stellen die Gliafärbung nicht ein Positivbild des Herdes ergab, sondern nur den in der Marksubstanz gelegenen Teil des Herdes sichtbar machte, so daß dieser im Gliabild gar nicht auf die Rinde überzugreifen schien." Für eine Deutung dieser eigenartigen Differenz in dem Verhalten des Stützgerüstes an Markund an Rindenherden erinnert $O$ ppenheim an die Tatsache, daß ,,in den Ganglienzellenschichten der Hirnrinde die Mehrzahl der Gliazellen normalerweise keine Fasern bilden und daß es nach Alzheimer erst bei verhältnismäßig schwerer Schädigung der nervösen Elemente hier zu pathologischer Faserbildung kommt. Es scheint also mit dem der multiplen Sklerose zugrunde liegenden Krankheitsprozeß keine derart schwere Schädigung der nervösen Strukturen einherzugehen, daß dadurch die normaliter faserlosen Gliazellen der Hirnrinde zu besonders reichlicher Faserbildung angeregt würden, im Gegensatz zu den Gliazellen der weißen Substanz und auch der grauen Substanz des Rückenmarks, die ja alle bereits in der Norm reichliche Faserbildung erkennen lassen und daher auf denselben Prozeß durch Bildung kompakter Gliawucherungen reagieren."

Ich habe mich auch jetzt wieder an einem größeren Material von multipler Sklerose und besonders an einigen sehr interessanten Fällen, die ich Herrn Oberarzt Dr. Nonne und dem Direktor der Heilanstalt Emmendingen Herrn Geheimrat Dr. Haardt verdanke, ausführlich mit den Rindenherden beschäftigt. Abgesehen von dem Material, das Gustav Oppenheim in unserem Laboratorium verarbeitete, fand ich auch jetzt an meinen neuen Fällen seine Angaben durchaus bestätigt. An dem Fehlen einer herdförmigen Gliafaserwucherung in den lediglich corticalen Herden ist nicht zu zweifeln. Auch darin stimmt also das Verhalten der Rindenherde bei Paralyse mit solchen bei multipler Sklerose durchaus überein - ein Umstand, der nicht zum wenigsten eben aus der eigenartigen Gliaverteilung in der Großhirnrinde zu erklären ist.

Nur in der Gestalt der Herde scheinen nicht unwesentliche Unter- 
schiede bei einem. Vergleich der Markscheidenbilder von multipler Sklerose und progressiver Paralyse zu bestehen. Bei den gewöhnlichen Fällen von multipler Sklerose sind ja die Großhirnherde häufig viel ausgedehnter, sie betreffen überhaupt vorwiegend das Mark und schieben sich von dort aus in die Rinde vor. Ihre Grenzen sind auch dort fast immer allseitig scharf und der ganze Herd hat im Markscheidenpräparat etwas in sich Abgeschlossenes. Der fleckige Markfaserschwund der Paralyse zeigt sich dagegen im allgemeinen aus kleinen Herden und unscharf begrenzten Lichtungen und Streifen zusammengesetzt. Aber

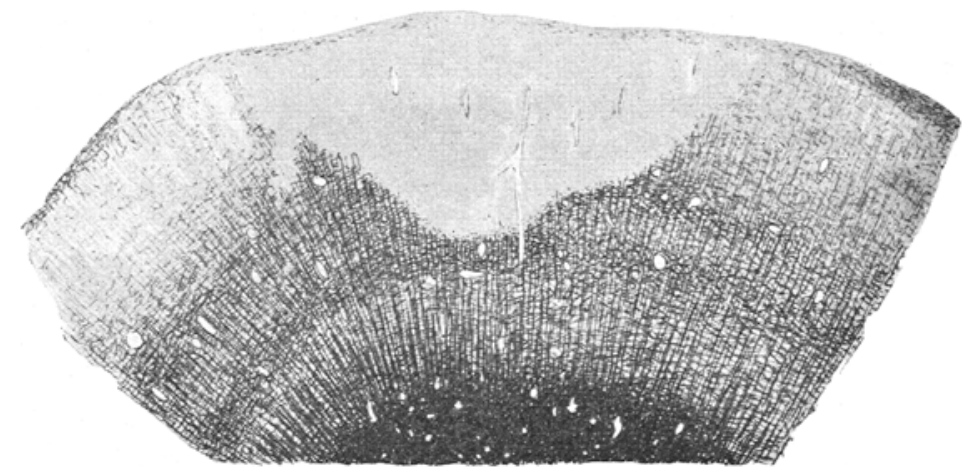

Fig. 6. Kleiner dreieckiger Herd in der oberen Rinde. Multiple Sklerose - Fall st. (Markscheidenfärbung am Gefrierschnitt.)

auch nur im allgemeinen. Denn wir sehen vielfach scharf aus der gesund erscheinenden Umgebung herausgeschnittene, völlig marklose Herde. Die Radiärfaserung ist oft wie durch einen Messerschnitt an ihrem Ursprung abgetragen. Und was das Wichtigste ist: es gibt Fälle von multipler Sklerose, in denen jene großen, aus der weißen Substanz in das Rindengrau reichenden Herde nur sehr selten sind, wo dagegen zahlreiche kleine Flecken und Lichtungen über die Meynertschen Schichten verstreut sind. Ich habe von dem in dieser Beziehung besonders charakteristischen Fall, den ich Herrn Oberarzt Dr. Nonne verdanke, hier eine Abbildung beigegeben (Taf. XII, Fig. 12), ebenso auch von einem anderen Falle mit vielen kleinen corticalen Herden (Fig. 6). Man trifft in diesen beiden Fällen auf sehr viele Rindenbilder, in denen es mir nahezu ausgeschlossen erscheint, daß auf Grund des Markscheidenbildes allein eine Entscheidung, ob Paralyse (mit starkem, fleckigem Markschwund) oder multiple Sklerose, getroffen werden könnte. In diesen Fällen und in vereinzelten Windungsbezirken anderer multipler Sklerosen weicht also auch die Gestaltung der Herde von der bei der Paralyse nicht ab.

Ich erwähnte aus der Arbeit Gustav Oppenheims, daß bei 
einem die Rinde und Mas Mark gleichzeitig treffenden Skleroseherde insofern eine wesentliche Differenz zwischen dem Rinden- und dem Markanteil desselben besteht, als $n$ ur von letzterem ein Positivbild mit dieser Färbung gewonnen werden kann. Wenn nun wirklich die marklosen Flecke bei der progressiven Paralyse den Rindenherden der multiplen Sklerose anatomisch nahe verwandt sind, so müßte auch an den Windungen, wo ausnahmsweise einmal ein markloser Herd in der paralytischen

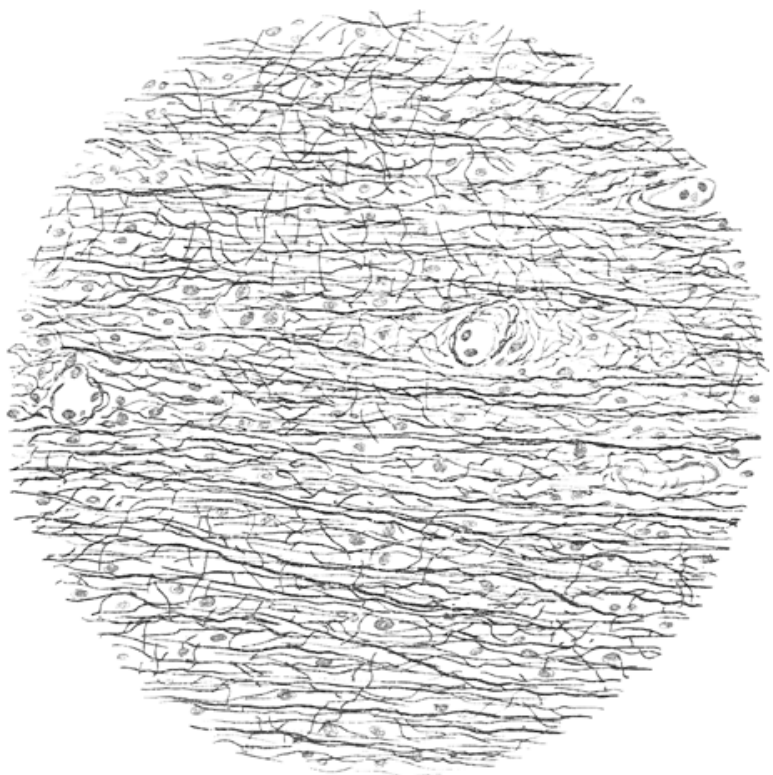

Fig. 7. Achsenzylinderpräparat (Bielschowsky). Ausschnitt aus dem in Fig. 11 bzw. Fig. 13 (Tafel XII bzw. XIII) dargestellten Herde, entsprechend der in Fig. 11 mit $x$ bezeichneten Partie.

Rinde tief in die weiße Substanz selbst hinübergreift, eine lokal begrenzte dichtfasrige Wucherung der Glia in dem Markanteil des Plaques nachzuweisen sein.

Und das ist tatsächlich der Fall. Natürlich nicht bei den Rindenherden, welche nur wenig über die Wurzeln der Radiärfaserung hinausgreifen; denn sie halten sich immer noch mit ihrer unteren Grenze in der tiefen Rinde (Taf. VIII, Fig. 4 u. Taf. X, Fig. 7). Wie bei gleichgelegenen Herden der multiplen Sklerose, findet man auch dort nur eine geringe lokale Vermehrung der Spinnenzellen, keine kompakte Gliawucherung in Herdform (vgl. auch Fischer). Dagegen treffen wir auf eine solche in den tief in die Marksubstanz selbst reichenden Herden (Taf. XI u. XII, Fig. 9-11). Bei zweien meiner Paralysen habe ich an je zwei Windungen derartige Herde gefunden. Bei 
der vergleichenden Untersuchung an Gefrierschnitten, die nach der Markscheidenfärbung bzw. nach der Achsenzylinder- und Neurogliamethode hergestellt waren, ließ sich an derim Mark gelegenen Partie des Herdes kein lokaler Ausfall von Achsenzylindern, vielmehr deren Persistenz erweisen, und es ließ sich weiter eine ko m pak te dichtfaserige Gliawucherung feststellen. Ich habe das in den Fig. 7; Taf. XII, Fig. 11 u. Taf. XIII, Fig. 13 abgebildet. Die eigenartige Gestalt jenes Herdes im Markscheidenbild wird wohl aus dem Photogramm ohne weiteres klar. Die adventitiellen Lymphräume der Gefäße enthalten im Bereiche des Herdes und seiner Umgebung mit Mark beladene Körnchenzellen, sie treten deshalb als schwarze Streifen auf dem Photogramm hervor. Das Positiv dieses Herdes gibt, wie das Ubersichtsbild auf Taf. XIII zeigt, das Neurogliapräparat. Die Grenzen dieser massigen Gliawucherung decken sich mit denen des Herdes am Markfaserbilde, sie erscheinen im Utbersichtsbild scharf wie die der gewöhnlichen Skleroseherde. Gegen die Rinde hört ebenfalls die Gliawucherung ziemlich plötzlich auf, wie wir das ja auch von den Skleroseplaques her kennen. In dem kernarmen dichten Glia filz finden wir auch große Gliazellen mit breiten, protoplasmatischen faserführenden Fortsätzen, ähnlich wieder wie gerade bei den Großhirnherden der multiplen Sklerose.

Der Einwand, es handle sich hier um eine histopathologisch und genetisch ganz andere Herdbildung als bei den gewöhnlichen Rindenflecken der Paralyse, würde nicht stichhaltig sein. Denn es fehlen alle Anzeichen für eine etwa durch regressive Gefäßveränderungen oder durch (syphilitische) Entzündungsvorgänge bewirkte lokale Schädigung; und vor allem zeigt ja das Achsenzylinderbild in dem Markteil des scharf begrenzten Herdes keine Reduktion der marklosen Fasern, verglichen mit der Fasermenge in dem intakten Teile des Radius.

In dem Markanteil des Herdes fanden sich Transportzellen, vereinzelt im Gewebe und zahlreich in den adventitiellen Lymphräumen; sie sind, wie gesagt, mit Markbestandteilen beladen, die sich bei der Markscheidenmethode schwarz färben; das Scharlachpräparat zeigt eine Anfüllung der Zellen auch mit lipoiden Produkten. Die im Gewebe liegenden Körnchenzellen sind sicher größtenteils gliogener Natur. Auch bei der multiplen Sklerose finden wir besonders in den jungen Herden solche Transportzellen verstreut und in den Lymphräumen der Gefäße aufgesammelt; und hier wie dort trifft man sie vorwiegend nur in der dem Mark angehörigen Partie eines Herdes, während in dem Rindenanteil eines solchen nur vereinzelte oder gar keine Körnchenzellen gelegen sind. Das erklärt sich offenbar aus dem gleichen Grunde, den Alzheimer für die Tatsache geltend macht, daß man die meisten und größten amöboiden Ciliazellen bei vielen Krankheitsvorgängen besonders 
in der Markleiste der Windungen trifft: es liegt das wohl daran, da $B$ das zerfallende Mark mehr fettige Produkte bei seinem Abbau liefert als die Rinde.

In der Vergleichung des fleckigen Markfaserschwundes bei Paralyse mit den Herden der multiplen Sklerose lege ich auf diese histologischen Feststellungen an den in das Mark vordringenden Herden den Hauptnachdruck. Die Seltenheit solcher Paralyseherde ändert nichts an ihrer prinzipiellen Bedeutung. Es soll ja nicht etwa bewiesen werden, daß die marklosen Herde der Par:lyse häufig zur Verwechslung mit den Rindenbildern der multiplen Sklerose Anlaß geben könnten; das wäre sinnlos; die Unterschiede zwischen beiden sind so bekannt und ergeben sich wohl auch ohnehin aus dieser Schilderung, daß wir darüber kein Wort zu verlieren brauchen. Es kommt hier vielmehr auf den Beweis an, daß der fleckige Markfaserschwund mit den Herden der multiplen Sklerose lediglich der rein histologischen Art nach nahe verwandt ist. Das aber ergibt sich ganz besonders auch aus den Befunden, an den Rindenmarkherden.

Wie diese somit die histologische Eigenart des rein corticalen fleckigen Markschwundes in der paralytischen Rinde klären, so erscheinen sie uns auch von maßgebender Bedeutung für die Auffassung der sklerotischen Herde im Rückenmark jenes Falles, von welchem wir hier ausgingen. Die histologische Utbereinstimmung jener Herde mit den Plaques der multiplen Sklerose überrascht uns danach nicht mehr in dem Maße wie anfangs. Wenn bei der Paralyse den Skleroseherden durchaus ähnliche Bildungen an der Grenze der Rinde zum Mark beobachtet werden, so kann uns das Vorkommen solcher Herde im Rückenmark nicht mehr als etwas prinzipiell Neues imponieren. Und wir haben keine zwingenden Gründe mehr, in diesem Fall unsere Zuflucht in der Annahme einer Kombination von progressiver Paralyse mit multipler Sklerose zu suchen (s. o.). Gerade die in diesem Fall so ausgesprochene und über das gewöhnliche Maß hinausgehende Neigung des paralytischen Prozesses, die corticalen Markgeflechte in Herd- und Fleckenform zu vernichten, macht es gewiß höchst wahrscheinlich, daß die zentrale paralytische Erkrankung es selbst ist, welche auch im Rückenmark den herdförmig begrenzten Markausfall in einigen wenigen Segmenten bewirkte. Daß sich diese Plaques durch die Markfaserwucherung von den Rindenherden unterscheiden müssen, ist nach dem Gesagten selbstverständlich.

Ich habe in dieser Arbeit zu zeigen versucht, daß in dem histologischen Gesamtbild der progressiven Paralyse einem fleckweise verteilten Ausfall der Markfaserung besondere Bedeutung zukommt und daß sich gerade in dieser Eigentümlichkeit des herdförmigen Mark- 
schwundes anatomische Ähnlichkeiten zwischen der Paralyse und der multiplen Sklerose ergeben. An der großen Häufigkeit des fleckigen Markschwundes in der paralytischen Rinde kann nicht mehr gezweifelt werden. Utber die Hälfte der Fälle von Paralyse zeigen in verschiedener Ausdehnung und Anordnung diesen eigenartigen Markausfall. Den markleeren Herden entspricht kein herdförmiger Ausfall von Achsenzylindern. Das Zell- und Gliabild zeigt dort lokal keine besonderen histologischen Eigentümlichkeiten. Das gleiche gilt für die Gefäße. Greift dagegen ein Herd - was sehr selten ist - auf die kompakte weiße Substanz über, so wird dort der Markausfall durch eine dichtfaserige Gliawucherung gedeckt; in frischen Herden finden sich in den dem Marklager angehörigen Teilen des Plaques fett- und markbeladene Körnchenzellen; die Achsenzylinder persistieren auch in solchen Herden.

Ich halte es für höchstwahrscheinlich, daß in sehr seltenen Fällen auch im Rückenmark diese Art eines fleckweise begrenzten Markschwundes zu Herden führen kann, die charakterisiert sind durch die Unabhängigkeit von irgendwelchen vasculären Prozessen, und die nicht mit Systemveränderungen in Zusammenhang stehen, die scharf gegen die Umgebung abgegrenzt sind, und die vor allem wieder eine Persistenz der Achsenzylinder neben einer dichtfaserigen Gliawucherung zeigen.

Es gibt also bei der progressiven Paralyse zwei Arten des Markfaserausfalls in der Rinde: erstens den gewöhnlichen, mehr gleichmäßigen Faserschwund und zweitens einen lokal akzentuierten, bei dem es zur Bildung markloserFlecke und Streifen, zu einer lokal beschränkten Entmarkung der Rinde und zu Veränderungen kommt, die ich kurzweg als ,corticalen Markfra B" bezeichnet habe, weil die in die Rinde strebenden derben Markfaserbündel und die corticalen Markfasergeflechte wie abgefressen erscheinen. Außer den echten Skleroseplaques gleichen diese marklosen Flecke in der paralytischen Rinde keinen anderen herdartigen Veränderungen. Von Entzündungsherden und Narbenbildungen sind sie wohl unterschieden.

$\mathrm{Daß}$ sie ein wichtiges anatomisches Charakteristikum der progressiven Paralyse bedeuten, folgt nicht nur aus der Häufigkeit ihres Vorkommens, sondern auch daraus, daß a naloge Bild ungen bei anderen chronischen Rindenerkrankungen nicht vorzukommen scheinen. Ich wage jedoch noch nicht zu behaupten, daß dem fleckigen Markschwund eine anatomisch differentialdiagnostische Bedeutung für die Paralyse zukommt (Fischer). Möglich ist das allerdings; denn auch wir haben in zahlreichen Markscheidenuntersuchungen bei den verschiedenartigsten organischen Psychosen und speziell bei groben Rindenkrankheiten analoge Herde nicht finden 
können. Auch bei der der Paralyse sonst so nahestehenden Schlafkrankheit bin ich ähnlichen Bildern nicht begegnet; ich habe allerdings nur 7 Fälle untersuchen können. Es scheint also, daß der corticale Markfaserschwund bei anderen chronischen schweren Rindenerkrankungen neben diffusen gleichmäßigen nicht auch herdartig begrenzte Ausfälle bewirkt, wie er das bei der progressiven Paralyse häufig tut. Und das würde allerdings für die anatomische Differentialdiagnose der Paralyse besonders wichtig sein und die Bedeutung des fleckigen Markschwundes für das histologische Gesamtbild der progressiven Paralyse noch wesentlich erhöhen. Aber erst ausgedehnte Kontrolluntersuchungen können darüber Aufschluß bringen.

Diese sind auch für die Klärung der weiteren Frage erforderlich: ob den Fällen mit ausgesprochenem herdförmigen Markschwund besondere klinische Eigentümlichkeiten zukommen, ob sie sich von den anderen Paralysen im Symptomenbild und Verlauf unterscheiden. Ich habe selbstverständlich auch in meinem Material darauf geachtet, und besonders in den 5 Fällen mit sehr intensivem, fleckigem Markfaserschwund nach speziellen klinischen Zügen gesucht. Mit negativem Erfolg. Schon die Mitteilung des Falles, von dem hier in erster Linie die Rede war, zeigte, daß es sich um eine ganz gewöhnliche Paralyse handelte. Stelle ich die anderen 4 Fälle dazu, so weisen auch sie nichts Eigenartiges auf, und sie unterscheiden sich wieder nicht unwesentlich von diesem und auch voneinander im psychischen und körperlichen Symptomenbild und nach Krankheitsdauer, Verlauf, Endzustand, Lebensalter usw. Auch Auftreten und Häufigkeit von Anfällen steht in keiner erkennbaren Korrelation zu der Häufigkeit und Verbreitung der Rindenherde. Es ist wohl zwecklos, hier die Krankengeschichten der betreffenden Fälle mitzuteilen.

Nur ein Fall hat ein gewisses Interesse, weil er der einzige war, der anfangs lange Schwierigkeiten in der differentialdiagnostischen klinischen Abgrenzung gegenüber einer multiplen Sklerose bot. Der Fall stammt noch aus der "Vor-Wassermannsehen Zeit", und die Lumbalpunktion konnte aus äußeren Gründen nicht gemacht werden; dadurch war die Diagnose besonders erschwert. Der betreffende Patient erkrankte unter dem Symptomenbild einer spastischen Spinalparalyse, verbunden mit Nystagmus, Intentionstremor, zitternder Sprache, Schwindel. Der spastische Symptomenkomplex verstärkte sich allmählich, der Nystagmus dagegen schwand, die Sprachstörung wurde schlimmer, behielt aber lediglich das Zittern im Klang (kein Schmieren, Silbenstolpern usw.). Allmählich entwickelte sich eine erhebliche psychische Schwäche; am Ende war der Patient, nach ca. $2 \frac{1}{2}$ jähriger Krankheitsdauer, schwer blödsinnig, dabei meist euphorisch, manchmal 
mehr depressiv oder erregt. Zuletzt hatte er rechtsseitige Zuckungen. Dieser Fall gehört zu den fünf mit starker Ausprägung des fleckigen Markschwundes; letzterer war besonders erheblich in der vorderen und hinteren Zentralwindung. Aber am Hemisphärenmark, Hirnstamm und Rückenmark wurden nirgends sklerotische Herde gefunden; der histologische Befund entsprach durchaus dem einer gewöhnlichen Paralyse. Der paralytische Prozeß hatte jedoch hier die Zentralwindungen in besonders intensiver Weise ergriffen; auf die degenerative Erkrankung und Zerstörung zahlreicher Riesenpyramidenzellen und anderer motorischer Rindenzellen in der vorderen Zentralwindung mußte die doppelseitige Pyramidenbahnerkrankung bezogen werden. Die Herde hatten die Eigenart, wie die Rindenherde bei der Paralyse sonst. Eine sekundäre Degeneration bewirkten sie nicht.

Aber wenn auch in diesem Fall der Befund von zahlreichen kleinen Herden in vielen Rindengebieten und speziell in den Zentralwindungen scheinbar gut $\mathrm{zu}$ seinen klinischen Eigentümlichkeiten und seiner symptomatologischen Ähnlichkeit mit einer multiplen Sklerose paßt, so sehen wir doch wieder andere Fälle, in denen wie hier fleckige Ausfälle im Markscheidenbild auch der Zentralwindungen gefunden werden, und in denen doch das klinische Bild keine der multiplen Sklerose gleichenden Züge trug. Also auch in dieser Richtung muß eine Fortführung dieser Untersuchungen Genaueres zu ermitteln suchen.

Und das gilt endlich noch für die anatomisch so sehr interessierende Frage: welche Eigenschaften das Ersatzgewebe hat, das in der Rinde an die Stelle des ausgefallenen Marks tritt, und wie sich ein Positiv für die Herde und Lichtungen des Markscheidenbildes färberisch gewinnen läßt. Ich hob ja schon hervor; $\mathrm{da} ß$ ich in dieser Beziehung nicht recht weiter gekommen bin, selbst mit den neuen Methoden Alzheimers nicht: Denn die Feststellung von einer nur gerade erkennbaren Vermehrung faserbildender und einfacher Gliazellen, die nach den Alzhei merschen Methoden bisweilen dargestellt werden kann und auch im Weigertschen Gliapräparat ab und $\mathrm{zu}$ angedeutet ist, kann ja nicht befriedigen. Trotzdem werden es gewiß Alzheimers neue Gliauntersuchungen und Studien über die Abbauvorgänge im zentralen Gewebe sein, die uns a uch hier den richtigen Weg weisen. Daß wir nicht schon heute einen klaren Einblick in jene histopathologischen Dinge bekommen, kann kaum wundernehmen, da eben das Rindengewebe bei seiner ungemeinen Kompliziertheit der anatomischen Analyse außerordentliche Schwierigkeiten bereitet. Sobald die Herde ausnahmsweise einmal über die tiefe Rinde hinaus in die weiße Substanz greifen, werden die Verhältnisse einfacher. Die gliöse Faserwucherung zeigt uns dort den Ersatz für 
die zugrunde gegangene Marksubstanz an, und mitunter finden wir in Körnchenzellen noch die Reste des zerfallenen Markes.

Gerade andiesen histopathologisch einfach zu beurteilenden Herdenglaubeich die anatomische Ähnlichkeitzwischen dem fleckigen Markschwund bei der Paralyse und den Herden der multiplen Sklerose bewiesen zu haben. Denn für die Behauptung einer histologischen Ubereinstimmung der lediglich in der Rinde gelegenen Herde bei der Paralyse mit ebensolchen bei multipler Sklerose konnten ja - neben den Analogien in Gestalt, Abgrenzung, Beziehung der Markfasern zur Umgebung usw. - eigentlich nur negative Momente geltend gemacht werden. Ich meine das Fehlen von korrespondierenden Herdveränderungen an anderen Elektivpräparaten. Andererseits ist natürlich gerade das Achsenzylinderbild in Anbetracht des Fehlens herdförmiger Veränderungen von prinzipieller Wichtigkeit, da sich eben daran die Persistenz der Achsenzylinder erweisen läßt. Wie die marklosen Flecke in der paralytischen Rinde, sind auch die auf die Rinde beschränkten Herde der multiplen Sklerose einer histologischen Analyse überaus schwer zugänglich. Bei ihnen gelang es ebenfalls nicht, ein Positiv für die Plaques am Markscheidenpräparat zu finden. Und es muß deshalb auch für die corticalen Plaques der multiplen Sklerose mit den neuen noch weiter zu vervollkommnenden Methoden eine histologische Differenzierung erstrebt werden, um die sich bereits Gust. Oppenheim mit seiner Methode $^{1}$ ) bemüht hat.

Gerade aber darin stimmt der fleckige Markfaserschwund bei der Paralyse mit den Herden der multiplen Sklerose überein, daß die Situation einfacher wird, wenn der Herd über die Rindengrenzen hinaus ins Mark dringt, weil eben dann eine dichtfaserige Gliawucherung den Markteil des Herdes kennzeichnet. Das scheint mir, wie gesagt, das wichtigste Beweismoment für die nahen anatomischen Beziehungen zwischen den marklosen Herden bei progressiver Paralyse und den Skleroseherden zu sein.

Spätere Untersuchungen werden vor allem nach solchen sich in das Mark ausdehnenden Herden zu fahnden haben. Auch die großen Ganglien, der Hirnstamm und das Kleinhirn müssen nach solchen Herden genauer durchsucht werden. Wir haben dort bisher, an einem allerdings unzureichenden Material, nichts gefunden. Daß dort überhaupt Herde vorkommen, ist bekannt (s. o.); es muß aber ermittelt werden, ob darunter auch solche sind, die prinzipiell mit den eben geschilderten Herden und also auch mit den Skleroseplaques übereinstimmen. Am Rückenmark kommen - wie ich an meinem

1) Gustar Oppenheim, Über protoplasmatische Gliastrukturen. Neurol. Centralbl. 1908. S. 643 . 
allerdings wohl ungemein seltenen Fall zeigen konnte - Herde vor, die einerseits ganz denen der multiplen Sklerose gleichen und deren prinzipielle Verwandtschaft mit den paralytischen Rindenherden andererseits meines Erachtens durch jene Herde höchstwahrscheinlich gemacht wird, die das Grenzgebiet zwischen Rinde und Mark einnehmen und deren histologische Eigentümlichkeiten ja hier genugsam betont wurden.

Es war der Zweck dieser Ausführungen, nur die Ähnlichkeit des herdförmigen Markschwundes bei der progressiven Paralyse und der multiplen Sklerose darzulegen. Das Interesse an den anatomischen Beziehungen zwischen multipler Sklerose und progressiver Paralyse ist damit nicht entfernt erschöpft. Im Gegenteil: es gewinnen diese anatomischen Beziehungen der Herde eine viel weittragendere Bedeutung durch die Tatsache, daß a uch bei der multiplen Sklerose die adventitiellen Gefäßräume im zentralen Nervensystem und die Meningen häufig beträchtliche Plasmazelleninfiltrate zeigen, und da $\beta$ auch hier - worauf ich schließlich noch hinweisen möchte - ein diffuser Markfaserschwund in der Rinde neben den eigentlichen Skleroseplaques nicht selten zur Beobachtung kommt (Taf. XII, Fig. 12).

In letzter Zeit ist von der Häufigkeit der Plasmazellinfiltrate bei der multiplen Sklerose mehrfach, wenn auch immer noch auffallend selten, die Rede gewesen, und besonders Gust. Oppenheim hat sich um die Erforschung dieser infiltrativen Vorgänge bemüht. Ich selbst kann an meinem Material Oppenheims Angaben durchaus bestätigen. Gerade auch in den beiden Fällen, auf die ich vorhin hingewiesen hatte, waren diese infiltrativen Prozesse besonders ausgesprochen. Für das histologische Gesamtbild der multiplen Sklerose ist in dieser Richtung noch mancherlei von Wichtigkeit, speziell erfordert die Frage noch ein eingehenderes Studium, ob nicht überhaupt Plasmazellinfiltrate bei der multiplen Sklerose regelmäßig, wenn auch oft nur spärlich, vorkommen, und ferner die Frage, wie es mit den Beziehungen zwischen Herden und Plasmazelleinlagerungen, mit der Verbreitung der vasculären und meningealen Infiltrate usw. steht. Das scheint schon jetzt, soviel ich sehe, sicher, daß neben den infiltrativen Vorgängen in den Plaques und ihrer Umgebung a uch mehr diff use Plasmazelleinlagerungen bei der multiplen Sklerose im Zentralorgan und speziell in der Rinde und ihrer Pia vorkommen.

Der diffuse Markfaserschwund in der Rinde bei multipler $\mathrm{Sklerose}$ ist bisher nicht gewürdigt worden. Und doch kann an dessen Häufigkeit gerade bei alten Fällen von multipler Sklerose mit groben psychischen Störungen nach meinen Beobachtungen nicht gezweifelt werden. Ich habe mich davon in einer ganzen Reihe von Fällen bei 
Anwendung meiner Markscheidenfärbung am Gefrierschnitt und der sonst gebräuchlichen Markscheidenmethoden überzeugen können. Die empfindlichen supraradiären und tangentialen Fasern leiden bei der multiplen Sklerose häufig ganz erheblich Not (Fig. 20). Sie sind bei manchen Windungen sehr stark gelichtet oder ganz geschwunden; es kann sich da nicht um Zufälligkeiten oder technische Mängel handeln.

Diese Dinge erfordern eine ausführliche Untersuchung und Beschreibung und lassen zusammen mit der Wegrichtung, welche Alzheimers jüngste Studie über die pathologische Neuroglia und die Abbauvorgänge gibt, das anatomische Studium der multiplen Sklerose von neuem aussichtsvoll erscheinen. Hier soll davon nicht noch gehandelt werden. Ich wollte nur deshalb darauf hinweisen, weil eben die Ähnlichkeit zwischen den Herdbildungen bei der progressiven Paralyse und der multiplen Sklerose mit Rücksicht auf diese histopathologischen Eigentümlichkeiten noch an Interesse gewinnt.

Wir sehen also, daß in der anatomischen Differentialdiagnose der progressiven Paralyse auch die multiple Sklerose eine wichtige Rolle spielt. Den skleroseähnlichen Herden kommt im histologischen Gesamtbild der progressiven Paralyse eine hervorragende Bedeutung zu. Und für die vergleichende Krankheitsforschung ist von besonderem Interesse, daß zwei dem Wesen und speziell der Ätiologie nach so verschiedene Krankheiten, wie die progressive Paralyse und die multiple Sklerose, in mehrfacher Hinsicht übereinstimmende histopathologische Züge aufweisen.

\section{Tafelerklärung ${ }^{1}$ ).}

Tafel VII und VIII Hirnrindenbilder vom Fall D (Paralyse).

Tafel VII.

Fig. 1. Fleckung der Rinde durch multiple marklose Herde. Im Zentrum der meisten Herde ein Gefäß. (Hintere Zentralwindung. Kulschitzkypräparat.)

Fig. 2. Auf der Kuppe der Windung ist die Ausstrahlung des Radius an ihrer Wurzel scharf abgeschnitten (,,corticaler Markfraß"); daneben kleinere Herde. (Stirnlappen. Kulschitzkypräparat.)

\section{Tafel VIII.}

Fig. 3. Großer, aus kleinen Herden entstandener markloser Bezirk. (Parietallappen. Markscheidenfärbung am Gefrierschnitt.)

Fig. 4. Markloser Fleck auf der Spitze einer Windung, der sich als scharf begrenztes, gelichtetes Feld in den Radius fortsetzt. (Parietallappen. Kulschitzkypräparat.)

1) Herr Professor Alzheimer hatte die Güte, die Herstellung der Photogramme im Laboratorium der Psychiatrischen Klinik in München zu besorgen. 


\section{Tafel IX.}

Fig. 5. Fall Cl. Paralyse. Völlig marklose Rinde; die Markfasern sind am Ursprunge des Markfächers ,abgeschnitten“. (I. Frontalwindung. Kul. schitzkypräparat.)

Fig. 6. Fall Kr. Paralyse. Der Calcarinastreifen ist auf eine große Strecke durch den Markscheidenschwund ausgelöscht. Am Zellpräparate erkennt man in dieser Partie deutlich den Calcarinatypus, ebenso am Bielschowskypräparat. Am Photogramm sieht man in dem markleeren Rindengebiet den lichten Streifen der Lamina ganglionaris $(x)$.

\section{Tafel X.}

Fig. 7. Fall K. Paralyse. Ein scharf begrenzter Herd nimmt die Windungskuppe etwa zur Hälfte ein; die andere Rindenhälfte erscheint noch auffallend faserreich. Der Herd greift etwas auf die kompakte Masse des Radius über. (Markscheidenfärbung am Gefrierschnitt.)

Fig. 8. Fall K. Paralyse. Zahlreiche marklose Flecken, zum Teil in Konfluenz. "Mottenfraßartige" Lichtungen in der Rindenfaserung. (Markschøidenfärbung am Gefrierschnitt.)

\section{Tafel XI.}

Fig. 9. Fall N. Paralyse. Großer markloser Rindenherd, der in zwei Zapfen auf die weiße Substanz übergreift. (Parietallappen. Kulschitzkypräparat.)

Fig. 10. Fall N. Paralyse. Großer, markloser Herd, der eine buchtartige Aushöhlung der kompakten Markmasse des Radius bewirkt. Im Bereiche der Gefäße $g$ Körnchenzellen. (Der Herd ist dem in Fig. 9 dargestellten benachbart. Kulschitzkypräparat.)

\section{Tafel XII.}

Fig. 11. Fall N. Paralyse. Tief in die weiße Substanz vordringender Herd, welcher vom Radius nur noch einen kleinen Stumpf (mit einem davon abgetrennten, seitlich gelegenen Streifen Marksubstanz) stehen läßt. Dieses Markscheidenpräparat entspricht dem Gliabilde der Tafel XIII. Von der mit $x$ bezeichneten Partie des Herdes stammt das Achsencylinderbild der Textfigur 7. G mit roten Blutkörperchen angefüllte Gefäße; in deren Bereiche Körnchenzellen. (Markscheidenfärbung am Gefrierschnitt.)

Fig. 12. Multiple Sklerose. Fall R. Multiple kleine Herde in der tiefen Rinde; daneben diffuser Schwund in den Markfasergeflechten der oberen Rinde. (Kulschitzkypräparat.)

\section{Tafel XIII.}

Neurogliafaserpräparat. U̇bersichtsbild. Fall N. Paralyse. Das Gliabild gibt das Positiv zu dem Markscheidenpräparat der Taf. XII, Fig. 11. Die Gliafaserwucherung, welcher in diesem Übersichtsbilde der blaugefärbte Bezirk entspricht, betrifft nur den Markanteil des Herdes. (Gefrierschnitt.) 